\title{
7. MOBILITY COMPARISONS: DOES USING DIFFERENT MEASURES MATTER?
}

\author{
Daniele Checchi and Valentino Dardanoni
}

\begin{abstract}
In this paper we review alternative measure of intergenerational mobility, emphasizing the distinction between absolute, relative and ordinal mobility. We then compare the performance of various mobility indices using real data. From Treiman and Ganzeboom (1990) dataset we compare the degree of occupational and educational intergenerational (father-son) mobility in 16 countries in a single year (comprised between 1968 and 1982). From three Bank of Italy surveys (1993, 1995, 1998) we obtain a comparable measure of social prestige and we show that intergenerational mobility in Italy across regions or age cohort exhibits different trends according to different indicators. We suggest that ordinal relative and absolute measures provide divergent indications whenever we compare mobility data with markedly different marginal distributions.
\end{abstract}

\section{INTRODUCTION}

When discussing mobility issues, a basic distinction is usually made between intergenerational and intragenerational mobility. The first concept concerns the study of how the distribution of some relevant measure of individual status

Inequality, Welfare and Poverty: Theory and Measurement, Volume 9, pages 113-145.

Copyright $\odot 2003$ by Elsevier Science Ltd.

All rights of reproduction in any form reserved.

ISBN: 0-7623-1014-6 
changes between different generations in a given society. Alternatively, intragenerational mobility studies how the distribution of individual status changes among a group of individuals over a given period of their lifetime.

In general, the simplest framework to capture either of these aspects is to consider how, in a society of $n$ individuals, a vector $x=\left(x_{1}, \ldots, x_{n}\right)$ is transformed into another vector $y=\left(y_{1}, \ldots, y_{n}\right)$, where $x_{i}$ denotes the value of a relevant observable indicator of the social and economic status of individual $i$, and $y_{i}$ denotes its value in the next generation (intergenerational case) or in the next time period (intragenerational case). Typical variables employed in most mobility studies for measuring socio-economic status are income, consumption, education, and occupational prestige. Henceforth, we will focus on intergenerational mobility and follow the usual convention of analyzing father to son movements in status as unit of analysis. Thus, the vector $x$ will describe the marginal distribution of status amongst the fathers and $y$ the marginal distribution of status amongst the sons in the society.

It is widely believed that socioeconomic mobility is somewhat an elusive concept, difficult to define, let alone to measure. This is in stark contrast with the literature on income inequality, where a consensus has emerged on what concepts of inequality mean, the correct theoretical procedures to measure them, and how to go from theory to empirical application. Mobility data $(x, y)$ describe the joint distribution of fathers' and sons' statuses in a population, while the vectors $x$ and $y$ describe their marginal distributions. In general, mobility data contain information about many different aspects of the mobility in a society. For instance, $x$ and $y$ each describe both the average level of status and its dispersion respectively within fathers and sons. Thus, one could say that that the marginal distributions contain information of a static nature. Mobility, on the other hand, concerns how the distribution of fathers' statuses $x$ is transformed into that of the sons $y$. Sociologists have suggested that, when analyzing mobility data, the interplay between the distributions of $x$ and $y$ can be described by two quite different concepts.

Structural mobility refers to how far apart $x$ is from $y$. For example, if a country is experimenting a substantial economic growth, there will be a greater number of high status positions available to the sons than there were for the fathers, and thus it determines some kind of social change. However, it is important to notice that there are many ways in which a given vector $y$ can be obtained from another vector $x$. In particular, two hypothetical societies could display the same amount of structural mobility because they have the same marginal distributions, but they could differ in how families interchange their relative positions. This second aspect is called exchange mobility by 
sociologists and refers to the positive association between fathers and sons statutes in the society.

Given the multifaceted nature of mobility data, we expect that mobility comparisons are intrinsically much more problematic than inequality comparisons. In particular, when analyzing the distribution of a single relevant variable in a population, as described by a real valued vector, we can typically summarize much of the information by two summary statistics on location ("the size of the pie") and dispersion ("the equality of its distribution"). On the other hand, when analyzing mobility data we need not only measures of location and dispersion both for the $x$ and the $y$ variables, but also summary statistics on the distance between the marginal distributions $x$ and $y$ (structural mobility) and their positive association (exchange mobility). Thus, we expect that comparing mobility data by a single summary mobility index may give results, which are very dependent on the characteristics of the chosen index, and we expect that the conclusions reached by the mobility analysis are more dependent on the choice of the mobility index when comparing societies with very different marginal distributions.

\section{MOBILITY INDICES}

To make our study manageable and the interpretation of the results consistent, in this paper we compare the performance of various mobility indices that are built up by aggregating the change in status occurring in each family in the society. Let us assume that family $i$ th has observed status indicators $\left(x_{i}, y_{i}\right)$. As a first methodological issue, we should consider whether $\left(x_{i}, y_{i}\right)$ describe accurately the concept of mobility that we want to capture. Let $h\left(x_{i} ; x\right)$ and $k\left(y_{i} ; y\right)$ denote real valued functions of observed status, monotonically increasing in $x_{i}$ and $y_{i}$ respectively, such that $h\left(x_{i} ; x\right)$ and $k\left(y_{i} ; y\right)$ capture what the researcher feels is "true" status of family $i$. For example, if $x$ and $y$ are the vectors of incomes in the population, the researcher may feel that income shares $\frac{x_{i}}{\bar{x}}$ and $\frac{y_{i}}{\bar{y}}$ (where $\bar{x}$ and $\bar{y}$ denote the means of $x$ and $y$ ) rather than incomes $x_{i}$ and $y_{i}$ are better indicators of family $i$ th status. If we feel that income shares capture the concept of mobility that we want to compare, then in the transition from $x$ to $y$, family $i$ has experienced a degree of mobility which is a function of the distance between $\frac{x_{i}}{\bar{x}}$ and $\frac{y_{i}}{\bar{y}}$. In general, let $d\left(h\left(x_{i} ; x\right), k\left(y_{i} ; y\right)\right)$ denote the numerical value taken by an appropriate distance function between true status $h\left(x_{i} ; x\right)$ and $k\left(y_{i} ; y\right)$ for family $i$. The function $d: \mathfrak{R}^{2} \Rightarrow \Re$ thus measures the 
degree of mobility at the family level. The class of mobility indices $M(x, y)$ that we will consider in this paper then simply aggregates all family distances $d\left(h\left(x_{i} ; x\right), k\left(y_{1} ; y\right), \ldots, d\left(h\left(x_{n} ; x\right), k\left(y_{n} ; y\right)\right)\right.$ by taking the average value:

$$
M(x, y)=\frac{1}{n} \sum_{i=1}^{n} d\left(h\left(x_{i} ; x\right), k\left(y_{i} ; y\right)\right)
$$

The class of mobility indices $M(x, y)$ is sufficiently rich to capture many widely employed indices. It is conceptually very simple, because it makes explicit that social mobility is simply an aggregation of family mobility, and depends on the explicit choice of the "transformation functions" $h$ and $k$ and the distance function $d$. Thus, $M(x, y)$ is sufficiently rich to capture many different views about the appropriate way of measuring mobility, since the researcher has simply to specify the functional form of $d, h$ and $k$ to derive a suitable index of mobility. In particular, depending on the choice of $h$ and $k, M(x, y)$ contains three subclasses of mobility indices: ${ }^{1}$

(1) Absolute indices: in this case the data $x$ and $y$ are directly employed to define true social status.

(2) Relative indices: we can distinguish between weakly relative indices, which are invariant to multiplication of $x$ and $y$ by common positive constant, strongly relative indices, which are invariant to multiplication of $x$ and $y$ by two possibly different positive constants, and affine indices which are invariant to possibly different linear transformations of $x$ and $y$.

(3) Ordinal indices: indices that are invariant to any monotonic transformation of the data. For example, any rank-based index is ordinal.

Two mobility indices that belong to $M(x, y)$ have been proposed in two important papers by Fields and Ok $(1996,1999)$. In the first of these papers Fields and Ok axiomatize a mobility index that takes $h$ and $k$ to be the identity function (thus observed status equal true status), and uses Euclidean distance for $d$ :

$$
M_{1}(x, y)=\frac{1}{n} \sum_{i=1}^{n}\left|y_{i}-x_{i}\right|
$$

In a recent paper, D'Agostino and Dardanoni (2002) axiomatize a class of mobility indices which lets $d\left(h\left(x_{i} ; x\right), k\left(y_{i} ; y\right)\right)=\left(h\left(x_{i} ; x\right)-k\left(y_{i} ; y\right)\right)^{2}$ and discuss 
various choices of the transformation functions $h$ and $k$. By letting $h$ and $k$ be again the identity function we have the index

$$
M_{2}(x, y)=\frac{1}{n} \sum_{i=1}^{n}\left(y_{i}-x_{i}\right)^{2}
$$

$M_{1}$ and $M_{2}$ are the absolute mobility indices considered in this paper.

Moving on to relative indices, Fields and Ok (1999) axiomatize an index that takes $h$ and $k$ to be the natural logarithm function, while still using Euclidean distance:

$$
M_{3}(x, y)=\frac{1}{n} \sum_{i=1}^{n}\left|\ln \left(y_{i}\right)-\ln \left(x_{i}\right)\right|
$$

On the other hand, taking income shares in D'Agostino and Dardanoni's class we get the index:

$$
M_{4}(x, y)=\frac{1}{n} \sum_{i=1}^{n}\left(\frac{y_{i}}{\bar{y}}-\frac{x_{i}}{\bar{x}}\right)^{2}
$$

We notice now that by appropriate choice of the functional form of $d, h$ and $k$, Pearson's correlation coefficient $\rho_{x y}$ is ordinally equivalent to an index in the class $M(x, y)$. In particular, letting $d\left(h\left(x_{i} ; x\right), k\left(y_{i} ; y\right)\right)=\left(h\left(x_{i} ; x\right)-k\left(y_{i} ; y\right)\right)^{2}$, we have that if $h\left(x_{i} ; x\right)=\frac{x_{i}-\bar{x}}{\sigma_{x}}$ and $k\left(y_{i} ; y\right)=\frac{y_{i}-\bar{y}}{\sigma_{y}}$ (the standardized values of $x_{i}$
and $\left.y_{i}\right)$

$$
M_{5}(x, y)=\frac{1}{2 n} \sum_{i=1}^{n}\left(\frac{x_{i}-\bar{x}}{\sigma_{x}}-\frac{y_{i}-\bar{y}}{\sigma_{y}}\right)^{2}
$$

and it can be shown that $M_{5}(x, y)=\left(1-\rho_{x y}\right)$. Clearly $M_{3}$ is weakly relative, $M_{4}$ is strongly relative and $M_{5}$ is affine.

Finally, ordinal indices are typically obtained by using ranks for defining true social status $h$ and $k$. Our next mobility index is thus

$$
M_{6}(x, y)=1-\lambda(x, y)=1-\frac{6}{n^{2}(n-1)} \sum_{i=1}^{n}\left(r\left(x_{i} ; x\right)-r\left(y_{i} ; y\right)\right)^{2}
$$

where $r\left(x_{i}, x\right)$ indicates the rank of $x_{i}, r\left(y_{i} ; y\right)$ indicates the rank of $y_{i}$ and $\lambda(x$, $y$ ) denotes the well-known non-parametric index of association of Spearman 
(Kendall \& Gibbons, 1990). However, while ranks are uniquely determined in the case where there are no ties in the marginal distributions, there is no single accepted way of defining ranks in the presence of ties. Spearman's $\lambda$ utilizes midranks for ranking tied values. On the other hand, if we use the cumulative distribution functions $F$ and $G$ to define family ranks for $x$ and $y$ respectively, we get an alternative ordinal index:

$$
M_{7}(x, y)=\frac{1}{n} \sum_{i=1}^{n}\left(F\left(x_{i}\right)-G\left(y_{i}\right)\right)^{2}
$$

while if we use Euclidean distance we get the index:

$$
M_{8}(x, y)=\frac{1}{n} \sum_{i=1}^{n}\left|F\left(x_{i}\right)-G\left(y_{i}\right)\right|
$$

Notice that $M_{6}$ is ordinally equivalent to $M_{7}$ whenever there are no ties in the marginal distributions and the populations we are comparing have equal size. ${ }^{2}$

In the following sections we will study how the eight indices considered above behave when used with some real datasets. As reference, we will also calculate two widely used indices of mobility, namely functions of the ordinary least square (OLS) regression coefficient when regressing $y$ on $\alpha+\beta x$ or $\log (y)$ on $\alpha+\beta \log (x)$ :

$$
\begin{aligned}
& M_{9}(x, y)=1-O L S_{y, x}=1-\frac{\sum_{i=1}^{n}\left(y_{i}-\bar{y}\right)\left(x_{i}-\bar{x}\right)}{\sum_{i=1}^{n}\left(x_{i}-\bar{x}\right)^{2}}=1-\frac{\sigma_{x}}{\sigma_{y}} \rho_{y x} \\
& M_{10}(x, y)=1-O L S_{l y, l x}=1-\frac{\sum_{i=1}^{n}\left(\ln y_{i}-\ln \bar{y}\right)\left(\ln x_{i}-\ln \bar{x}\right)}{\sum_{i=1}^{n}\left(\ln x_{i}-\ln \bar{x}\right)^{2}}
\end{aligned}
$$

It can be easily verified that $M_{9}$ is weakly relative while $M_{10}$ is strongly relative.

In Sections 3 and 4 we will apply the ten mobility indices above to two real datasets. We expect that absolute indices will be the most sensitive to differences in marginal distributions, while ordinal indices will be the less sensitive. In fact, if we are comparing two mobility data without ties in the marginal distributions, ordinal indices, by taking ranks, are calculated on transformed variables with identical marginal distributions regardless of the shape of the original distributions. On the other hand, if we are comparing two mobility data which differ for the extent of socioeconomic growth between the fathers and sons generation, absolute indices will always display a greater level of mobility in presence of greater growth even if in both societies there is a perfect positive association between fathers' and sons' statuses (that is, there is 
no exchange mobility). Thus, we expect that ordinal indices will give greater weight to the exchange component of mobility, while absolute indices will give greater weight to the structural component. Notice however that ordinal indices will be the more sensitive to differences in marginal distributions the greater the extent of tied values, depending on the choice of the status transformations $h$ and $k$.

Finally, notice that relative indices fall somewhat in between absolute and ordinal ones; depending on the choice of the transformations $h$ and $k$ performed to raw data, relative indices may reduce the influence of differences in the marginal distributions in differing fashions. In general, structural mobility may have resulted from many different sources (generalized proportional growth; alternatively status changes might have been concentrated only in higher or lower levels classes; or there could have been substantial changes in inequality, etc.). Thus, taking shares, logs, differences from average values, standardized differences, etc. will reduce the effect of differences in marginal distributions thus giving less weight to structural mobility, for a given level of exchange mobility. The extent of this reduction will be dependent on the chosen transformations $h$ and $k$.

\section{A FIRST EMPIRICAL APPLICATION}

The first empirical exercise applies to the 10 measures of mobility considered above to an international comparison. Treiman and Ganzeboom (1990) have collected data on occupational mobility from 31 different surveys conducted in 16 countries $^{3}$ over a period of 14 years (from 1968 to 1982). This dataset is composed only of men and contains information about the respondent age, marital status, educational achievement (both as type of degree and in terms of year), his current occupation (coded under alternative classifications), working hours, supervisorship role and self-employment condition. Self-reported current earnings and actual family incomes (measured in local currency) are also available, but in some cases they are reported at intervals, thus rendering cross-country comparisons almost impossible. Moreover, the dataset lacks direct information about father incomes. Finally, information on education and occupation of father, mother and spouse are also available. Treiman and Ganzeboom (1990) provide a consistent ordering of occupations for crosscountry comparisons, based on social prestige. Two alternative measures of social prestige are available: the ISEI - international status of employment index (ranging between 0 and 90) and the TREI index (ranging between 0 and 86), originally proposed by Treiman (1977). Both measures are strongly 
correlated with respondent age, income and years of education (see Table 2). Given the high correlation between the two indices $(0.75$ over the entire sample), we will report results for the former index only. Table 1 contains information about sample size and averages for education, income and relative rank positions for both respondents and their fathers; the same table also displays Gini indices for each marginal distribution.

There are two variables in this dataset that can be used to analyze intergenerational mobility: (occupational) social prestige and years of education. In Tables 3 and 4 we report the value of the 10 mobility indices considered in this paper both for occupational and educational mobility and also the ranking of the mobility data according to the 10 indices. The last column in both tables gives the overall ranking obtained by averaging the rank under all the indices. Note that there are 31 mobility data for the case of occupational mobility while only 29 for the case of educational mobility, since the data on father's education are missing for Brazil 1973 and Northern Ireland 1968.

We notice that U.S., Taiwan and the Netherlands come out consistently as the most mobile societies, both in terms of occupation and education-based mobility. It is rather surprising to find that Germany under different surveys comes out as the least mobile society in terms of educational achievements mobility.

We next compute the correlation matrix of the 10 indices across different surveys. A glance at Table 5 reveals that a very different picture emerges in the two cases of occupation and education-based mobility comparisons. In particular, the correlations between the 10 mobility indices are generally much higher using occupational prestige rather than years of education as variables.

These different positive correlations in the two cases of occupational and educational mobility can be explained by various hypotheses. In general, while occupational mobility tracks changes in the productive structure, such that we record a generalized improvement in the average "quality" of jobs but with possibly a high variance among different groups, educational mobility is enhanced mainly during the process of mass access to education, given that compulsory education forces the young generation to obtain a given amount of schooling. Thus, in general we expect that the difference in inequality between the marginal distributions of $x$ and $y$ is lower for occupation rather than education. This is confirmed by looking again at Table 1, where we have calculated the Gini coefficient for the marginal distributions in the two cases. We notice that there is a decline in inequality of educational achievement, but not in occupational prestige.

However, the most plausible explanation of the much greater correlation between the various indices when considering occupational rather than 


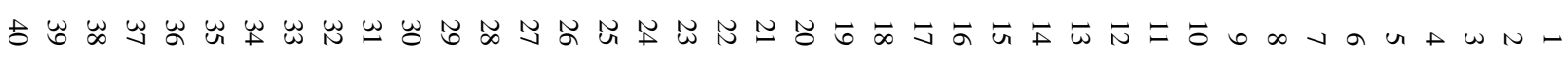

Table 1. Observations Available for Cross-Country Comparisons - Sample Averages.

\begin{tabular}{|c|c|c|c|c|c|c|c|c|c|c|c|c|}
\hline Country & $\begin{array}{l}\text { Survey } \\
\text { year }\end{array}$ & $\begin{array}{l}\text { Survey } \\
\text { label }\end{array}$ & $\begin{array}{c}\text { Number } \\
\text { observations }\end{array}$ & $\begin{array}{l}\text { Respondent } \\
\text { personal } \\
\text { income (local } \\
\text { currency) }\end{array}$ & $\begin{array}{c}\text { Respondent } \\
\text { occupational } \\
\text { prestige } \\
\text { (ISEI) }\end{array}$ & $\begin{array}{l}\text { Respondent } \\
\text { father } \\
\text { occupational } \\
\text { prestige } \\
\text { (FISEI) }\end{array}$ & $\begin{array}{l}\text { Respondent } \\
\text { years of } \\
\text { education } \\
\text { (educyr) }\end{array}$ & $\begin{array}{l}\text { Respondent } \\
\text { father years } \\
\text { of education } \\
\text { (feducyr) }\end{array}$ & $\begin{array}{c}\text { Gini index } \\
\text { respondent } \\
\text { occupational } \\
\text { prestige }\end{array}$ & $\begin{array}{l}\text { Gini index } \\
\text { respondent } \\
\text { father } \\
\text { occupational } \\
\text { prestige }\end{array}$ & $\begin{array}{l}\text { Gini index } \\
\text { respondent } \\
\text { years of } \\
\text { education }\end{array}$ & $\begin{array}{l}\text { Gini index } \\
\text { respondent } \\
\text { father years } \\
\text { of education }\end{array}$ \\
\hline Australia & 1974 & AUT74P & 452 & 6872.48 & 40.58 & 35.11 & 9.96 & 7.25 & 0.204 & 0.193 & 0.100 & 0.124 \\
\hline Brazil & 1973 & BRA73 & 6743 & 1592.31 & 33.81 & 25.11 & 4.75 & - & 0.258 & 0.268 & 0.422 & - \\
\hline Brazil & 1982 & BRA82 & 8742 & 72.68 & 37.79 & 28.98 & 4.53 & 2.62 & 0.224 & 0.244 & 0.472 & 0.528 \\
\hline England & 1972 & ENG72 & 7027 & 1940.78 & 43.21 & 37.44 & 9.98 & 8.95 & 0.175 & 0.156 & 0.087 & 0.081 \\
\hline England & 1974 & ENG74P & 377 & 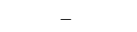 & 41.84 & 43.31 & 10.28 & 8.81 & 0.177 & 0.194 & 0.124 & 0.134 \\
\hline Finland & 1975 & FIN75P & 388 & 1605.14 & 38.91 & 32.27 & 8.94 & 7.79 & 0.176 & 0.200 & 0.151 & 0.128 \\
\hline Germany & 1975 & GER75P & 635 & 1572.52 & 44.77 & 39.09 & 9.70 & 8.03 & 0.178 & 0.200 & 0.119 & 0.083 \\
\hline Germany & 1976 & GER76Z & 503 & 1487.52 & 46.13 & 40.02 & 11.14 & 9.78 & 0.175 & 0.193 & 0.111 & 0.086 \\
\hline Germany & 1977 & GER77Z & 377 & 1816.01 & 44.55 & 39.68 & 10.64 & 9.87 & 0.178 & 0.191 & 0.122 & 0.072 \\
\hline Germany & 1978 & GER78W & 440 & 1999.37 & 42.55 & 39.32 & 10.38 & 9.93 & 0.164 & 0.190 & 0.123 & 0.092 \\
\hline Germany & 1979 & GER79X & 405 & 2010.42 & 45.34 & 39.59 & 10.73 & 9.76 & 0.173 & 0.182 & 0.127 & 0.085 \\
\hline Germany & 1979 & GER79Z & 441 & 2081.41 & 46.12 & 39.64 & 10.72 & 9.70 & 0.175 & 0.169 & 0.114 & 0.084 \\
\hline Germany & 1980 & GER80Z & 421 & 2264.12 & 46.55 & 39.21 & 10.78 & 9.66 & 0.207 & 0.237 & 0.169 & 0.248 \\
\hline Germany & 1980 & GER80a & 706 & 2176.40 & 44.63 & 38.91 & 10.37 & 9.70 & 0.170 & 0.153 & 0.652 & 0.784 \\
\hline Hungary & 1982 & HUN82 & 4745 & 469.65 & 38.48 & 31.46 & 9.74 & 7.25 & 0.217 & 0.202 & 0.128 & 0.141 \\
\hline Indonesia & 1971 & IND71 & 1980 & 138.94 & 41.40 & 41.83 & 3.18 & 1.75 & 0.173 & 0.170 & 0.280 & 0.312 \\
\hline Ireland & 1973 & IRE73 & 1807 & 1662.36 & 37.11 & 32.65 & $\begin{array}{l}70.36 \\
10.36\end{array}$ & 8.63 & 0.193 & 0.209 & 0.161 & 0.148 \\
\hline Italy & 1975 & ITA75P & 413 & & 41.07 & 33.93 & 7.75 & 4.96 & 0.189 & 0.186 & 0.169 & \\
\hline Japan & 1975 & JAP75 & 2271 & 2170.54 & 43.75 & 37.55 & 10.71 & 7.60 & 0.199 & 0.197 & 0.118 & 0.133 \\
\hline Netherlands & 1974 & NET74P & 350 & 1505.74 & 47.37 & 39.91 & 10.16 & 7.64 & 0.186 & 0.210 & 0.162 & 0.192 \\
\hline Netherlands & 1977 & NET77 & 1252 & 4.00 & 47.30 & 41.66 & 11.17 & 8.12 & 0.187 & 0.203 & 0.190 & 0.196 \\
\hline Netherlands & 1982 & NET82A & 309 & 574.99 & 46.91 & 41.75 & 10.02 & 8.40 & 0.185 & 0.192 & 0.175 & 0.194 \\
\hline Netherlands & 1982 & NET82B & 599 & 26454.49 & 48.83 & 44.45 & 11.06 & 9.04 & 0.168 & 0.191 & 0.122 & 0.186 \\
\hline Northern Ireland & 1968 & NIR & 430 & & 39.60 & 33.27 & 5.12 & - & 0.208 & 0.165 & 0.394 & 0.596 \\
\hline Northern Ireland & 1973 & NIR & 1876 & 1866.82 & 40.04 & 34.88 & 10.19 & 8.14 & 0.192 & 0.157 & 0.375 & 0.574 \\
\hline Philippines & 1968 & PHI & 6670 & 2573.69 & 35.23 & 31.80 & 7.41 & 3.85 & 0.185 & 0.192 & 0.152 & 0.146 \\
\hline Philippines & 1973 & PHI & 2468 & 3014.28 & 34.74 & 30.39 & 7.10 & 3.72 & 0.208 & 0.195 & 0.394 & 0.657 \\
\hline Switzerland & 1976 & SWI & 392 & 2938.79 & 44.55 & 36.93 & 9.31 & 7.79 & 0.186 & 0.183 & 0.067 & 0.073 \\
\hline Taiwan & 1970 & TAI & 990 & 36.48 & 41.08 & 35.67 & 5.12 & 5.36 & 0.187 & 0.138 & 0.096 & 0.083 \\
\hline United States & 1973 & USA & 26788 & 1125.91 & 44.07 & 37.20 & 11.82 & 8.36 & 0.206 & 0.219 & 0.145 & 0.275 \\
\hline \multirow[t]{2}{*}{ United States } & 1974 & USA & 432 & 13708.62 & 48.50 & 39.64 & 12.70 & 9.49 & 0.193 & 0.204 & 0.134 & 0.232 \\
\hline & & Total & 81429 & - & 39.31 & 37.18 & 9.08 & 6.92 & 0.190 & 0.193 & 0.199 & 0.230 \\
\hline
\end{tabular}




\begin{tabular}{|c|c|c|c|c|}
\hline $\begin{array}{l}\text { No. obs: } \\
\text { Depvar: }\end{array}$ & $\begin{array}{c}76402 \\
\text { trei }\end{array}$ & $\begin{array}{c}76402 \\
\text { isei }\end{array}$ & $\begin{array}{c}80207 \\
\text { trei }\end{array}$ & $\begin{array}{c}80207 \\
\text { isei }\end{array}$ \\
\hline \multirow[t]{2}{*}{ age } & 0.086 & 0.075 & 0.082 & 0.072 \\
\hline & (24.41) & (18.76) & (26.28) & $(23.11)$ \\
\hline \multirow[t]{2}{*}{ education/years } & 1.384 & 2.095 & 0.729 & 1.194 \\
\hline & (93.15) & (126.25) & $(55.91)$ & (86.46) \\
\hline $\begin{array}{l}\text { log prs } \\
\text { income }\end{array}$ & $\begin{array}{r}3.441 \\
(50.17)\end{array}$ & $\begin{array}{c}5.694 \\
(72.27)\end{array}$ & & \\
\hline $\begin{array}{l}\text { log median } \\
\text { occupation/income }\end{array}$ & & & $\begin{array}{r}13.527 \\
(126.13)\end{array}$ & $\begin{array}{r}21.689 \\
(176.35)\end{array}$ \\
\hline $\begin{array}{l}\text { Study } \\
\text { dummmies }\end{array}$ & Yes & Yes & Yes & Yes \\
\hline $\begin{array}{l}\text { Years } \\
\text { dummies }\end{array}$ & Yes & Yes & Yes & Yes \\
\hline $\mathrm{R}^{2}$ & 0.932 & 0.924 & 0.942 & 0.947 \\
\hline
\end{tabular}

educational mobility is entirely due to the different nature of the scale of measurement employed for the two variables. Occupational prestige is typically an ordinal scale, while a ratio scale measures education. Thus, data on occupational prestige are intrinsically less sensitive to the various transformations (shares, ranks, logs, etc.) required to obtain the 10 indices considered. On the other hand, years of educations take intrinsically fewer values than occupational prestige, so that there are many more tied values in the marginal distributions of education rather than occupation. Thus, for example, the ordinal indices $M_{6}$ and $M_{7}$ which are theoretically almost perfectly correlated in the case of no ties (in which case the indices are actually measuring pure exchange mobility) have greater correlation in the occupation rather than the education example.

Looking at Table 5, it also emerges that absolute, relative and ordinal mobility indices give quite different views of the degree of mobility present in the different data. For the reasons just explained, we will comment only on the correlation matrix for the education-based calculations, where the effect of the chosen transformations is clearer and more marked. We notice first that the two absolute indices $M_{1}$ and $M_{2}$ have correlation equal to 0.942 . On the other hand, there is much less agreement between the relative indices $M_{3}, M_{4}, M_{5}, M_{9}$ and 
$M_{10}$ : while the correlation between OLS coefficient calculated on education and its logarithm counterpart have correlation equal to $0.805, M_{3}$ has negative correlation $(-0.182)$ with the OLS coefficient, and low positive correlation (0.254) with the $\log$ OLS coefficient. Even more surprising is the strong negative correlation $(-0.718)$ between the two strongly relative indices $M_{4}$ and $M_{5}$. Given the generally changing level of inequality between the marginal

Table 3. Alternative Measures of Intergenerational Mobility - Occupational Social Prestige - Cross-Country Sample.

Country year index 1 index 2 index 3 index 4 index 5 index 6 index 7 index 8 index 9 index 10

\begin{tabular}{|c|c|c|c|c|c|c|c|c|c|c|c|}
\hline USA & 74 & 16.33 & 452.11 & 0.37 & 0.29 & 0.73 & 0.73 & 0.11 & 0.27 & 0.70 & 0.73 \\
\hline Netherlands & 82 & 1445 & 356.97 & 0.32 & 0.18 & 0.73 & 0.69 & 0.11 & 0.26 & 0.74 & 0.77 \\
\hline USA & 73 & 14.29 & 380.81 & 035 & 0.28 & 0.65 & 0.65 & 0.10 & 0.25 & 063 & 0.66 \\
\hline Hungary & 82 & 12.99 & 312.98 & 0.38 & 0.32 & 0.62 & 0.64 & 010 & 0.25 & 0.60 & 0.66 \\
\hline U.K. & 72 & 12.23 & 277.63 & 0.31 & 0.20 & 0.64 & 0.68 & 0.11 & 0.26 & 0.60 & 0.67 \\
\hline Germany & 77 & 12.30 & 295.74 & 0.29 & 0.19 & 0.63 & 0.68 & 0.11 & 0.26 & 0.64 & 0.67 \\
\hline Taiwan & 70 & 12.25 & 340.22 & 0.30 & 0.27 & 0.70 & 0.68 & 0.07 & 0.23 & 0.68 & 0.67 \\
\hline Finland & 75 & 10.88 & 221.97 & 0.29 & 0.21 & 0.66 & 0.72 & 0.11 & 0.27 & 0.57 & 0.65 \\
\hline Netherlands & 82 & 13.11 & 301.21 & 0.30 & 0.17 & 0.62 & 0.65 & 0.10 & 0.25 & 0.60 & 0.62 \\
\hline Japan & 75 & 12.48 & 319.72 & 0.31 & 0.23 & 0.61 & 0.62 & 0.09 & 0.24 & 0.62 & 0.63 \\
\hline Germany & 80 & 12.50 & 281.14 & 0.27 & 0.17 & 0.62 & 0.65 & 0.11 & 0.25 & 0.58 & 0.61 \\
\hline Germany & 80 & 11.66 & 263.92 & 0.30 & 0.18 & 0.62 & 0.64 & 0.10 & 0.25 & 0.60 & 0.65 \\
\hline Germany & 75 & 11.85 & 275.70 & 0.29 & 0.18 & 0.62 & 0.63 & 0.10 & 0.25 & 0.61 & 0.65 \\
\hline Brazil & 82 & 12.84 & 332.96 & 0.39 & 0.40 & 0.58 & 0.58 & 0.09 & 0.24 & 0.54 & 0.59 \\
\hline N. Ireland & 73 & 11.34 & 258.14 & 0.30 & 0.21 & 0.59 & 0.65 & 0.11 & 0.26 & 0.53 & 0.61 \\
\hline U.K. & 74 & 12.17 & 218.50 & 0.31 & 0.12 & 0.61 & 0.62 & 0.14 & 0.29 & 0.50 & 0.62 \\
\hline Italy & 75 & 11.00 & 232.61 & 0.30 & 0.20 & 0.63 & 0.62 & 0.09 & 0.25 & 0.57 & 0.60 \\
\hline N. Ireland & 68 & 10.79 & 235.12 & 0.29 & 0.21 & 0.59 & 0.64 & 0.10 & 0.25 & 0.53 & 0.62 \\
\hline Netherlands & 77 & 12.71 & 301.76 & 0.30 & 0.17 & 0.57 & 0.58 & 0.09 & 0.24 & 0.56 & 0.60 \\
\hline Germany & 78 & 10.65 & 247.86 & 0.26 & 0.16 & 0.61 & 0.63 & 0.10 & 0.23 & 0.62 & 0.66 \\
\hline Netherlands & 74 & 12.51 & 299.45 & 0.30 & 0.19 & 0.52 & 053 & 0.09 & 0.23 & 0.50 & 0.56 \\
\hline Germany & 78 & 11.83 & 262.39 & 0.28 & 0.17 & 0.57 & 0.61 & 0.10 & 0.25 & 0.57 & 0.60 \\
\hline Brazil & 73 & 11.78 & 295.76 & 0.39 & 0.47 & 0.50 & 0.52 & 0.07 & 0.23 & 0.46 & 0.50 \\
\hline Switzerland & 76 & 11.57 & 265.07 & 0.29 & 0.19 & 0.53 & 0.55 & 0.09 & 0.23 & 0.47 & 0.56 \\
\hline Ireland & 73 & 9.69 & 215.51 & 0.27 & 0.20 & 0.49 & 0.55 & 0.09 & 0.24 & 0.42 & 0.47 \\
\hline Germany & 76 & 11.35 & 248.84 & 0.27 & 0.16 & 0.47 & 0.56 & 0.09 & 0.23 & 0.49 & 0.55 \\
\hline Philippines & 73 & 8.00 & I85.89 & 0.22 & 0.20 & 0.60 & 0.58 & 0.07 & 0.22 & 0.53 & 0.54 \\
\hline Germany & 78 & 10.42 & 217.55 & 0.25 & 0.14 & 0.48 & 0.54 & 0.09 & 0.23 & 0.51 & 0.56 \\
\hline Australia & 74 & 10.19 & 221.99 & 0.26 & 0.18 & 0.50 & 0.50 & 0.08 & 0.23 & 0.43 & 0.44 \\
\hline Philippines & 68 & 8.41 & 185.30 & 0.24 & 0.18 & 0.53 & 0.52 & 0.07 & 0.21 & 0.44 & 0.44 \\
\hline Indonesia & 71 & 6.88 & 156.53 & 0.19 & 0.09 & 0.46 & 0.46 & 0.09 & 0.16 & 0.43 & 0.41 \\
\hline
\end{tabular}


Table 3. Continued.

\begin{tabular}{|c|c|c|c|c|c|c|c|c|c|c|c|c|}
\hline Country & yea & nk & ank & ank & ank & ank & ank & rank & rank & rank9 & rank & avg.ran \\
\hline USA & 74 & 31 & 31 & 28 & 28 & 30 & 31 & 29 & 29 & 30 & 30 & 30 \\
\hline Netherlands & 82 & 30 & 29 & 26 & 12 & 31 & 29 & 30 & 26 & 31 & 31 & 28 \\
\hline USA & 73 & 29 & 30 & 27 & 27 & 27 & 25 & 19 & 18 & 27 & 24 & 25 \\
\hline Hungary & 82 & 27 & 25 & 29 & 29 & 21 & 21 & 20 & 20 & 22 & 26 & 24 \\
\hline U.K. & 72 & 19 & 18 & 25 & 18 & 26 & 27 & 27 & 28 & 21 & 27 & 24 \\
\hline Germany & 77 & 21 & 20 & 13 & 15 & 24 & 26 & 28 & 27 & 28 & 28 & 23 \\
\hline Taiwan & 70 & 20 & 28 & 19 & 26 & 29 & 28 & 3 & 11 & 29 & 29 & 22 \\
\hline Finland & 75 & 9 & 7 & 14 & 24 & 28 & 30 & 26 & 30 & 16 & 21 & 21 \\
\hline Netherlands & 82 & 28 & 23 & 22 & 7 & 19 & 22 & 23 & 21 & 20 & 17 & 20 \\
\hline Japan & 75 & 22 & 26 & 23 & 25 & 18 & 15 & 9 & 13 & 25 & 20 & 20 \\
\hline Germany & 80 & 23 & 19 & 9 & 9 & 23 & 23 & 25 & 24 & 19 & 15 & 19 \\
\hline Germany & 80 & 14 & 15 & 17 & 13 & 22 & 19 & 21 & 23 & 23 & 22 & 19 \\
\hline Germany & 75 & 17 & 17 & 12 & 11 & 20 & 18 & 22 & 22 & 24 & 23 & 19 \\
\hline Brazil & 82 & 26 & 27 & 30 & 30 & 12 & 12 & 7 & 12 & 14 & 11 & 18 \\
\hline N. Ireland & 73 & 11 & 13 & 20 & 22 & 13 & 24 & 24 & 25 & 13 & 16 & 18 \\
\hline U.K. & 74 & 18 & 6 & 24 & 2 & 16 & 14 & 31 & 31 & 8 & 19 & 17 \\
\hline Italy & 75 & 10 & 9 & 16 & 20 & 25 & 16 & 14 & 17 & 17 & 13 & 16 \\
\hline N. Ireland & 68 & 8 & 10 & 15 & 23 & 14 & 20 & 18 & 19 & 12 & 18 & 16 \\
\hline Netherlands & 77 & 25 & 24 & 18 & 8 & 10 & 10 & 15 & 15 & 15 & 14 & 15 \\
\hline Germany & 78 & 7 & 11 & 5 & 5 & 17 & 17 & 17 & 7 & 26 & 25 & 14 \\
\hline Netherlands & 74 & 24 & 22 & 21 & 16 & 7 & 5 & 12 & 9 & 9 & 10 & 14 \\
\hline Germany & 78 & 16 & 14 & 10 & 6 & 11 & 13 & 16 & 16 & 18 & 12 & 13 \\
\hline Brazil & 73 & 15 & 21 & 31 & 31 & 6 & 3 & 2 & 5 & 5 & 5 & 12 \\
\hline Switzerland & 76 & 13 & 16 & 11 & 17 & 8 & 7 & 6 & 10 & 6 & 8 & 10 \\
\hline Ireland & 73 & 4 & 4 & 7 & 21 & 4 & 8 & 11 & 14 & 1 & 4 & 8 \\
\hline Germany & 76 & 12 & 12 & 8 & 4 & 2 & 9 & 10 & 6 & 7 & 7 & 8 \\
\hline Philippines & 73 & 2 & 3 & 2 & 19 & 15 & 11 & 4 & 3 & 11 & 6 & 8 \\
\hline Germany & 78 & 6 & 5 & 4 & 3 & 3 & 6 & 8 & 8 & 10 & 9 & 6 \\
\hline Australia & $74 v$ & 5 & 8 & 6 & 10 & 5 & 2 & 5 & 4 & 2 & 3 & 5 \\
\hline Philippines & 68 & 3 & 2 & 3 & 14 & 9 & 4 & 1 & 2 & 4 & 2 & 4 \\
\hline Indonesia & 71 & 1 & 1 & 1 & 1 & 1 & 1 & 13 & 1 & 3 & 1 & 2 \\
\hline
\end{tabular}

distributions of education of the fathers and the sons (Table 1), we expect that since $M_{5}$ is normalized by the standard deviation it would be less sensitive to changes in marginal distributions, thus behaving closer to ordinal indices rather than absolute ones. This expectation is confirmed by Table 5, where it emerges that $M_{4}$ seems to be positively correlated with the absolute indices and negatively correlated with ordinal ones, while $M_{5}$ has the opposite behavior. Regarding ordinal indices, it seems that while the choice of ranks in the presence of ties does make an important difference $\left(M_{6}\right.$ and $M_{7}$ have correlation 
of only 0.47 ), the choice of the family distance function (absolute value vs. squared difference) does not seem to make much practical difference. Finally notice that all ordinal indices seem to have positive (if in some cases moderate) correlation with all other indices except $M_{4}$.

This example shows rather dramatically that the choice of a mobility index has a substantial effect on the results, depending on the data used: when marginal distributions are different, each index gives a different weight to the inequality of the marginal distribution and to the structural and exchange component of overall mobility.

Table 4. Alternative Measures of Intergenerational Mobility - Years of Education - Cross-Country Sample.

\begin{tabular}{|c|c|c|c|c|c|c|c|c|c|c|c|}
\hline Country & yea & & $U_{\lambda}$ & laex & H & ndex & index & ndex & index & $d$ & d \\
\hline Taiwan & 70 & 4.73 & 42.19 & 0.66 & 1.47 & 0.60 & 0.52 & 0.07 & 0.21 & 0.77 & 0.88 \\
\hline USA & 74 & 3.92 & 26.00 & 0.38 & 0.29 & 0.54 & 0.58 & 0.10 & 0.25 & 0.63 & 0.76 \\
\hline USA & 73 & 4.10 & 27.13 & 0.44 & 0.39 & 0.52 & 0.53 & 0.09 & 0.23 & 0.63 & 0.74 \\
\hline N. Ireland & 73 & 2.39 & 10.73 & 0.26 & 0.16 & 0.60 & 0.64 & 0.11 & 0.26 & 0.56 & 0.68 \\
\hline Hungary & 82 & 3.30 & 18.11 & 0.39 & 0.34 & 0.57 & 0.55 & 0.08 & 0.22 & 0.61 & 0.66 \\
\hline Netherlands & 77 & 3.71 & 24.89 & 0.39 & 0.38 & 0.57 & 0.58 & 0.08 & 0.23 & 0.52 & 0.58 \\
\hline Netherlands & 82 & 2.99 & 15.27 & 0.33 & 0.22 & 0.63 & 0.66 & 0.07 & 0.20 & 0.65 & 0.69 \\
\hline Netherlands & 82 & 3.14 & 16.34 & 0.33 & 020 & 0.62 & $0 . \mathrm{GS}$ & $0 . \mathrm{OG}$ & 0.20 & 0.65 & 0.75 \\
\hline Ireland & 73 & 2.35 & 10.04 & 0.24 & 013 & 0.59 & 0.61 & 0.10 & 0.24 & 0.58 & 0.66 \\
\hline U.K. & 72 & 1.29 & 3.37 & 0.14 & 0.04 & 0.64 & 0.67 & 0.10 & 0.25 & 0.66 & 0.76 \\
\hline Philippines & 73 & 4.00 & 30.01 & 0.60 & 2.16 & 0.46 & 0.48 & 0.07 & 0.21 & 0.36 & 0.58 \\
\hline Switzerland & 76 & 1.94 & 9.32 & 0.24 & 0.15 & 0.57 & 0.58 & 0.09 & 0.24 & 0.51 & 0.69 \\
\hline Japan & 75 & 3.43 & 19.98 & 0.37 & 0.35 & 0.54 & 0.55 & 0.07 & 0.22 & 0.38 & 0.50 \\
\hline Germany & 76 & 2.20 & 13.80 & 0.20 & 0.14 & 0.60 & 0.61 & $0.0 \mathrm{G}$ & 0.19 & 0.53 & 0.59 \\
\hline Finland & 75 & 1.42 & 5.32 & 0.16 & 0.09 & 0.66 & 0.64 & 0.08 & 0.23 & 0.57 & 0.64 \\
\hline Netherlands & 74 & 2.94 & 14.63 & 0.34 & 0.25 & 0.45 & 0.54 & 0.08 & 0.22 & 0.45 & 0.57 \\
\hline Philippines & 68 & 4.19 & 31.32 & 0.65 & 2.12 & 0.38 & 0.39 & 0.06 & 0.19 & 0.27 & 0.55 \\
\hline Australia & 74 & 2.96 & 12.15 & 0.35 & 0.23 & 0.54 & 0.54 & 0.05 & 0.19 & 0.52 & 0.61 \\
\hline Italy & 75 & 3.30 & 21.24 & 0.55 & 0.87 & 0.45 & 0.39 & 0.05 & 0.18 & 0.32 & 0.45 \\
\hline Brazil & 82 & 2.68 & 14.40 & 0.59 & 2.10 & 0.41 & 0.40 & 0.06 & 0.19 & 0.14 & 0.45 \\
\hline Germany & 80 & 1.62 & 9.47 & 0.15 & 0.10 & 0.59 & 0.59 & 0.05 & 0.18 & 0.46 & 0.55 \\
\hline U.K. & 74 & 1.72 & 6.34 & 0.17 & 0.08 & 0.48 & 0.59 & 0.10 & 0.24 & 0.30 & 0.47 \\
\hline Germany & 75 & 2.06 & 8.08 & 0.23 & 0.13 & 0.47 & 0.47 & 0.07 & 0.20 & 0.46 & 0.51 \\
\hline Indonesia & 71 & 1.92 & 11.42 & 0.51 & 3.72 & 0.33 & 0.35 & 0.03 & 0.15 & 0.15 & 0.53 \\
\hline Germany & 78 & 1.52 & 8.68 & 0.13 & 0.09 & 0.54 & 0.56 & 0.04 & 0.16 & 0.42 & 0.51 \\
\hline Germany & 80 & 1.33 & 7.59 & 0.12 & 0.08 & 0.54 & 0.52 & 0.04 & 0.15 & 0.45 & 0.44 \\
\hline Germany & 78 & 1.29 & 7.40 & 0.11 & 0.08 & 0.56 & 0.54 & 0.02 & 0.12 & 0.49 & 0.49 \\
\hline Germany & 78 & 1.45 & 8.14 & 0.12 & 0.09 & 0.54 & 0.51 & 0.03 & 0.15 & 0.37 & 0.37 \\
\hline Germany & 77 & 1.33 & 7.76 & 0.11 & 0.08 & 0.51 & 0.54 & 0.02 & 0.12 & 0.38 & 0.42 \\
\hline
\end{tabular}


Table 4. Continued.

\begin{tabular}{|c|c|c|c|c|c|c|c|c|c|c|c|c|}
\hline Country & year & ank1 & rank2 & rank3 & rank4 & rank5 & rank6 & rank7 & rank8 & rank9 & rank10 & avg.rank \\
\hline Taiwan & 70 & 29 & 29 & 29 & 25 & 24 & 8 & 17 & 17 & 29 & 29 & 24 \\
\hline USA & 74 & 25 & 25 & 20 & 19 & 12 & 18 & 27 & 27 & 25 & 28 & 23 \\
\hline USA & 73 & 27 & 26 & 23 & 23 & 10 & 10 & 24 & 21 & 24 & 25 & 21 \\
\hline N. Ireland & 73 & 15 & 13 & 14 & 14 & 25 & 26 & 29 & 29 & 20 & 22 & 21 \\
\hline Hungary & 82 & 22 & 21 & 22 & 20 & 19 & 16 & 22 & 19 & 23 & 21 & 21 \\
\hline Netherlands & 77 & 24 & 24 & 21 & 22 & 18 & 20 & 20 & 23 & 18 & 15 & 21 \\
\hline Netherlands & 82 & 19 & 19 & 15 & 16 & 27 & 28 & 16 & 14 & 26 & 24 & 20 \\
\hline Netherlands & 82 & 20 & 20 & 16 & 15 & 26 & 27 & 11 & 13 & 27 & 26 & 20 \\
\hline Ireland & 73 & 14 & 12 & 13 & 11 & 22 & 24 & 25 & 24 & 22 & 20 & 19 \\
\hline U.K. & 72 & 2 & 1 & 6 & 1 & 28 & 29 & 28 & 28 & 28 & 27 & 18 \\
\hline Philippines & 73 & 26 & 27 & 27 & 28 & 6 & 6 & 15 & 16 & 6 & 16 & 17 \\
\hline Switzerland & 76 & 11 & 10 & 12 & 13 & 20 & 19 & 23 & 25 & 16 & 23 & 17 \\
\hline Japan & 75 & 23 & 22 & 19 & 21 & 11 & 15 & 18 & 18 & 8 & 8 & 16 \\
\hline Germany & 76 & 13 & 16 & 10 & 12 & 23 & 23 & 13 & 12 & 19 & 17 & 16 \\
\hline Finland & 75 & 5 & 2 & 8 & 7 & 29 & 25 & 19 & 22 & 21 & 19 & 16 \\
\hline Netherlands & 74 & 17 & 18 & 17 & 18 & 4 & 11 & 21 & 20 & 11 & 14 & 15 \\
\hline Philippines & 68 & 28 & 28 & 28 & 27 & 2 & 3 & 10 & 9 & 3 & 13 & 15 \\
\hline Australia & 74 & 18 & 15 & 18 & 17 & 14 & 12 & 8 & 11 & 17 & 18 & 15 \\
\hline Italy & 75 & 21 & 23 & 25 & 24 & 5 & 2 & 9 & 8 & 5 & 4 & 13 \\
\hline Brazil & 82 & 16 & 17 & 26 & 26 & 3 & 4 & 12 & 10 & 1 & 5 & 12 \\
\hline Germany & 80 & 8 & 11 & 7 & 9 & 21 & 22 & 7 & 7 & 14 & 12 & 12 \\
\hline U.K. & 74 & 9 & 3 & 9 & 5 & 8 & 21 & 26 & 26 & 4 & 6 & 12 \\
\hline Germany & 75 & 12 & 7 & 11 & 10 & 7 & 5 & 14 & 15 & 13 & 10 & 10 \\
\hline Indonesia & 71 & 10 & 14 & 24 & 29 & 1 & 1 & 3 & 3 & 2 & 11 & 10 \\
\hline Germany & 78 & 7 & 9 & 5 & 8 & 13 & 17 & 6 & 6 & 10 & 9 & 9 \\
\hline Germany & 80 & 4 & 5 & 3 & 4 & 16 & 9 & 5 & 5 & 12 & 3 & 7 \\
\hline Germany & 78 & 1 & 4 & 1 & 2 & 17 & 14 & 1 & 1 & 15 & 7 & 6 \\
\hline Germany & 78 & 6 & 8 & 4 & 6 & 15 & 7 & 4 & 4 & 7 & 1 & 6 \\
\hline Germany & 77 & 3 & 6 & 2 & 3 & 9 & 13 & 2 & 2 & 9 & 2 & 5 \\
\hline
\end{tabular}

\section{A SECOND EMPIRICAL APPLICATION}

We now move to the analysis of the Italian case. Differently from other countries, Italy does not possess a longitudinal survey that is long enough to provide information on actual incomes of both parents and children. ${ }^{4} \mathrm{~A}$ data set on intergenerational mobility based on occupational status has been built in 1985 by a group of sociologists from different Italian universities. ${ }^{5}$ A representative sample of 5016 individuals aged between 18 and 65 was interviewed about their working life and their social attitudes; additional 
Table 5. Correlation Between Different Measures of Mobility - Cross-Country Sample.

\begin{tabular}{|c|c|c|c|c|}
\hline & \multicolumn{4}{|r|}{ Occupational Prestige } \\
\hline & index 1 & index 2 & index 3 & index 4 index 5 index 6 index 7 index 8 index 9 index 10 \\
\hline index 1 & 1.0000 & & & \\
\hline index 2 & 0.9298 & 1.0000 & & \\
\hline index 3 & 0.7980 & 0.7820 & 1.0000 & \\
\hline index 4 & 0.3751 & 0.5173 & 0.8031 & 1.0000 \\
\hline index 5 & 0.6364 & 0.6238 & 0.4289 & 0.16481 .0000 \\
\hline index 6 & 0.6460 & 0.5707 & 0.4232 & 0.11090 .91851 .0000 \\
\hline index 7 & 0.4583 & 0.2182 & 0.1961 & -0.31950 .51170 .63121 .0000 \\
\hline index 8 & 0.6773 & 0.4408 & 0.5163 & 0.09340 .65190 .78400 .71991 .0000 \\
\hline index 9 & 0.7088 & 0.7229 & 0.4100 & $\begin{array}{llllll}0.1193 & 0.9029 & 0.8538 & 0.4491 & 0.5326 & 1.0000\end{array}$ \\
\hline \multirow[t]{3}{*}{ index 10} & 0.7735 & 0.6909 & 0.5070 & $\begin{array}{lllllll}0.1116 & 0.8780 & 0.9067 & 0.6238 & 0.7337 & 0.9332 & 1.0000\end{array}$ \\
\hline & \multicolumn{4}{|r|}{ Years of Education } \\
\hline & index 1 & index 2 & index 3 & index 4 index 5 index 6 index 7 index 8 index 9 index 10 \\
\hline index 1 & 1.0000 & & & \\
\hline index 2 & 0.9419 & 1.0000 & & \\
\hline index 3 & 0.8449 & 0.8318 & 1.0000 & \\
\hline index 4 & 0.3498 & 0.4337 & 0.7513 & 1.0000 \\
\hline index 5 & -0.1679 & -0.1891 & -0.5036 & -0.71791 .0000 \\
\hline index6 & -0.2301 & -0.3124 & -0.5934 & -0.74840 .89681 .0000 \\
\hline index 7 & 0.3476 & 0.1797 & 0.1270 & $-0.23470 .32640 .4701 \quad 1.0000$ \\
\hline index 8 & 0.3699 & 0.1984 & 0.1596 & -0.20040 .33360 .46050 .98431 .0000 \\
\hline index 9 & 0.2147 & 0.1745 & -0.1820 & - 0.54940 .82830 .73630 .46270 .44801 .0000 \\
\hline index 10 & 0.4719 & 0.4136 & 0.2541 & $\begin{array}{lllllll}-0.0584 & 0.5081 & 0.4796 & 0.6298 & 0.6351 & 0.8050 & 1.0000\end{array}$ \\
\hline
\end{tabular}

questions were asked about family background. From this file it is possible to extract information concerning the interviewed person referred to 1985 and referring to his/her family when he/she was 14 years old. As a consequence, the generation of sons is observed at the same time, whereas their parents are observed in different years, ranging in principle from 1934 to $1981 .^{6}$ This data set has been widely analyzed. ${ }^{7}$ International comparison indicates that Italy exhibits a lower degree of intergenerational mobility, both in terms of occupational characteristics (prestige or incomes) and educational achievements.

Another source of information on intergenerational persistence is provided by the Bank of Italy Survey on Household Incomes and Wealth (SHIW), 
conducted biannually since $1977 .{ }^{8}$ Given the panel component of this survey is rather limited, we have to rely on recall information about the parent status, which is available from the 1993 survey. From sociological literature (and in the absence of direct information about parent incomes) we accept the idea that occupations represent a good indicator of long run status achieved by a person. However, the SHIW data set does not provide a detailed classification of occupation, and therefore we cannot resort to an indicator of prestige, ${ }^{9}$ as we have done in the previous application. In addition, we prefer to stick to the economists' viewpoint that incomes are the best summary statistics available on the relative desirability of a social position. However we also know that educational achievement represents a rough measure of the human capital accumulated by an individual. Therefore we have resorted to rank individuals according to their earned income and their educational achievement. ${ }^{10}$ This implies that we assume that social ordering is substantially based on spending ability, which in turn derives from earned income and human wealth. In order to eliminate the erratic component based on individual fortunes, we consider the median income associated to any combination of job position and educational achievement, and we rank individuals accordingly.

In the absence of direct information about parent actual incomes, we cannot provide a generation specific ranking and we are forced to use the same ranking for both generations. One could object that each generation should possess its own ranking, which reflect events specific to that age cohort (degree of industrial development, wars, etc.). But data availability prevents this possibility, even if we are aware that part of the observed mobility is actually due to the process of development, the change in the distribution of occupations and the process of mass schooling. Similar methodology has been used by Checchi, Ichino and Rustichini 1999 to obtain measures of occupational status for the Italian case (see also Benabou \& Ok, 2001).

We make use of the SHIW surveys conducted in 1993, 1995 and $1998 .{ }^{11}$ It comprises 68.838 individuals, gathered into 23.371 families. Among the individuals, there are 41.753 individuals with a non-null income. Total net income is obtained from dependent labor employment, from self-employment, from pensions or from ownership of capital. Since income from selfemployment activity are plagued by under-reporting, ${ }^{12}$ we have revised it upward by $40 \%$, which corresponds to the discrepancy between post-tax income from self-employment and corresponding values based on national accounts (averaged over the period 1980-1993). For each member of the family we have information about his/her maximum educational achievement (but not about the educational career - we ignore any attendance without graduation), the current work status and the current or past sector of employment. In 
addition we have also analogous information about the parents of the household head and his/her spouse. This information is indicatively referred to the same current age of the respondent. ${ }^{13}$

In order to rank people according to their occupations, in addition to educational attainment we know the work status and the sector of employment of the interviewees. Unfortunately, the disaggregation of work status, sectors and educational achievements for parents is less detailed than the corresponding disaggregation for children. Therefore we have aggregated information about children in order to be comparable with the corresponding aggregation of their parents. By restricting to individuals who are employed and earn a positive income, we obtain 23,700 individuals in the children generation. The percentage distribution of relevant variables in the two generations is reported in Table 6.

By combining educational credentials (5 items), work status (8 items) and sector of employment (4 items), we get 160 potential combinations of these features, whereas actual combinations associated with non-negative incomes are only 122. For each cell identified by a combination of education/work status/sector we have computed the median and the mean income in the full sample. The orderings of all combinations is reported in Table 7, where one can notice that ranking according to the mean or to the median are rather similar, since the two measures are highly correlated. ${ }^{14}$ In order to define an index of social prestige, in the sequel we make use of the ranking based on median income. $^{15}$

Once we have introduced a cardinal measure of income that renders comparable two generations, we can analyze intergenerational mobility by calculating the 10 mobility indices above. We start by noticing first that inequality is higher in the parents generations than in the children generation, as grasped by Table 8: all inequality measures referred to the parent generation dominate the corresponding measures for the children generation. ${ }^{16}$ In addition, it is worth emphasizing that an ordinal measure of social position (reported in column 6 of Table 7 and corresponding almost completely to the rank associated with each combination education/work status/sector in an ordering based on median incomes) implies a degree of inequality which is closer to the inequality in actual incomes rather than median occupational incomes. In any case, by recording a lower inequality in social positions across generations we could anticipate that some "equalizer device" has operated along the century. Industrial development, implying significant reallocation of jobs among sectors and the emergence of new occupations and/or educational push are the best candidates to this explanation. 
We now move to the proper analysis of intergenerational mobility. Following a consolidated procedure, we consider the couple father-son, to avoid distortion due to differences in participation rates across generations and/or regions. ${ }^{17} \mathrm{We}$ make use of ten previously introduced indices, using either a territorial disaggregation or a birth cohort disaggregation. ${ }^{18}$

We start by considering mobility comparisons in different Italian regions. It is well known that Italy is characterized by a rather unequal distribution of

Table 6. Comparable Distributions Across Generations Italy 1993, 1995, 1998.

\begin{tabular}{|c|c|c|c|c|}
\hline Educational achievement & 1 & 2 & 3 & 4 \\
\hline no education & 1.34 & 1.59 & 23.66 & 27.48 \\
\hline primary school (elementare) & 14.78 & 18.29 & 51.5 & 4.34 \\
\hline lower secondary school (scuola media) & 33.1 & 33.2 & 13.52 & 10.9 \\
\hline upper secondary school (scuola superiore) & 39.09 & 35.55 & 8.08 & 6.16 \\
\hline bachelor (laurea) & 11.69 & 11.36 & 3.24 & 1.12 \\
\hline \multicolumn{5}{|l|}{ Work status } \\
\hline blue collar & 34.69 & 32.35 & 48.51 & 44.08 \\
\hline office worker & 27.05 & 26.16 & 13.96 & 8.24 \\
\hline teacher & 7.91 & 5.17 & 1.35 & 7.87 \\
\hline junior manager-official & 4.56 & 6.02 & 3.15 & 1.55 \\
\hline senior manager & 1.91 & 3.01 & 1.22 & 0.05 \\
\hline professional & 3.73 & 4.44 & 1.99 & 1.01 \\
\hline entrepreneur & 1.25 & 1.85 & 1.99 & 1.31 \\
\hline self-employed & 18.9 & 20.99 & 27.83 & 35.89 \\
\hline \multicolumn{5}{|l|}{ Sector of employment } \\
\hline agriculture & 4.68 & 4.48 & 24.44 & 36.62 \\
\hline industry & 32.1 & 33.13 & 22.94 & 14.93 \\
\hline public administration & 28.94 & 30.06 & 16.67 & 15.98 \\
\hline private services & 34.28 & 32.33 & 35.95 & 32.47 \\
\hline Number of cases & 23700 & 12187 & 11901 & 11913 \\
\hline
\end{tabular}

Legend:

$1=$ whole sample of employed in the generation of children

$2=$ household head sample of employed in the generation of children

$3=$ (employed $)$ father of (employed) household head

$4=$ (employed $)$ mother of (employed) household head 


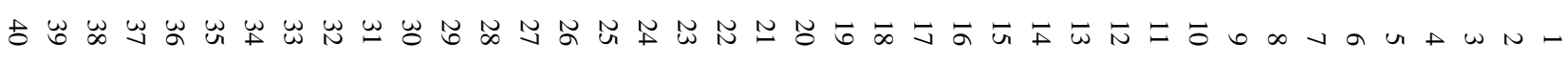

Table 7. Ordering of occupations - Italy 1993, 1995, 1998.

\begin{tabular}{|c|c|c|c|c|c|c|c|c|}
\hline Cases & $\begin{array}{c}\text { Median } \\
\text { income } \\
\text { (1998 euros) }\end{array}$ & $\begin{array}{l}\text { Rank } \\
\text { median }\end{array}$ & $\begin{array}{c}\text { Mean } \\
\text { income } \\
\text { (1998 euros) }\end{array}$ & $\begin{array}{l}\text { Rank } \\
\text { mean }\end{array}$ & $\begin{array}{l}\text { Rank } \\
\text { (final) }\end{array}$ & Education & Work status & Sector of activity \\
\hline 4 & 1936.713 & 1 & 2361.843 & 1 & 1 & primary & office worker & agriculture \\
\hline 45 & 5941.331 & 2 & 7800.203 & 4 & 2 & no educ & self-employed & private services \\
\hline 1 & 6197.483 & 3 & 6197.483 & 2 & 3 & no educ & entrepreneur & private services \\
\hline 70 & 6589.893 & 4 & 6779.366 & 3 & 4 & no educ & blue collar & agriculture \\
\hline 33 & 6916.473 & 5 & 10492.471 & 2 & 5 & no educ & self-employed & agriculture \\
\hline 193 & 7044.363 & 6 & 8386.782 & 7 & 6 & primary & blue collar & agriculture \\
\hline 4 & 7082.21 & 7 & 10304.361 & 1 & 7 & lower secondary & teacher & public administ \\
\hline 207 & 7381.662 & 8 & 8164.44 & 6 & 8 & lower secondary & blue collar & agriculture \\
\hline 34 & 7867.519 & 9 & 8644.603 & 8 & 9 & no educ & blue collar & private services \\
\hline 1 & 8068.865 & 10 & 8068.865 & 5 & 10 & no educ & office worker & industry \\
\hline 123 & 8781.226 & 11 & 11791.26 & 21 & 11 & lower secondary & self-employed & agriculture \\
\hline 18 & 8921.609 & 12 & 14061.07 & 33 & 12 & no educ & self-employed & industry \\
\hline 1 & 9037.996 & 13 & 9037.996 & 9 & 13 & primary & professional & industry \\
\hline 479 & 9296.225 & 14 & 10010.38 & 10 & 14 & upper secondary & blue collar & private services \\
\hline 20 & 9442.246 & 15 & 14547.45 & 36 & 15 & primary & entrepreneur & private services \\
\hline 63 & 9792.55 & 16 & 11329.22 & 16 & 16 & upper secondary & blue collar & agriculture \\
\hline 1139 & 9802.021 & 17 & 10541.86 & 13 & 17 & lower secondary & blue collar & private services \\
\hline 207 & 10032.12 & 18 & 13013.22 & 30 & 18 & primary & self-employed & agriculture \\
\hline 7 & 10140.12 & 19 & 10769.92 & 15 & 19 & bachelor & blue collar & private services \\
\hline 8 & 10601.62 & 20 & 10664.45 & 14 & 20 & bachelor & blue collar & industry \\
\hline 627 & 11120.28 & 21 & 14108.63 & 34 & 21 & primary & self-employed & private services \\
\hline 461 & 11127.82 & 22 & 11776.14 & 20 & 22 & primary & blue collar & private services \\
\hline 2392 & 11159.6 & 23 & 12216.92 & 24 & 23 & lower secondary & blue collar & industry \\
\hline 6 & 11219.3 & 24 & 12104.04 & 23 & 24 & lower secondary & office worker & agriculture \\
\hline
\end{tabular}




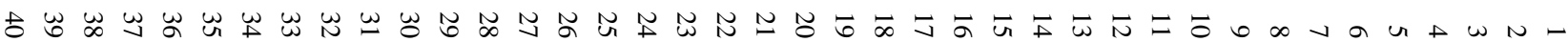

Table 7. Continued.

\begin{tabular}{|c|c|c|c|c|c|c|c|c|}
\hline Cases & $\begin{array}{c}\text { Median } \\
\text { income } \\
\text { (1998 euros) }\end{array}$ & $\begin{array}{c}\text { Rank } \\
\text { median }\end{array}$ & $\begin{array}{c}\text { Mean } \\
\text { income } \\
\text { (1998 euros) }\end{array}$ & $\begin{array}{l}\text { Rank } \\
\text { mean }\end{array}$ & $\begin{array}{l}\text { Rank } \\
\text { (final) }\end{array}$ & Education & Work status & Sector of activity \\
\hline 6 & 11302.35 & 25 & 11371.47 & 17 & 25 & bachelor & blue collar & public administ \\
\hline 97 & 11382.79 & 26 & 11452.44 & 18 & 26 & no educ & blue collar & industry \\
\hline 895 & 11489.42 & 27 & 12733.27 & 28 & 27 & upper secondary & blue collar & industry \\
\hline 1163 & 11578.14 & 28 & 14774.68 & 38 & 28 & lower secondary & self-employed & private services \\
\hline 1 & 12222.47 & 30 & 12222.47 & 25 & 29 & primary & teacher & public administ \\
\hline 9 & 12252.53 & 31 & 11644.44 & 19 & 30 & upper secondary & teacher & private services \\
\hline 1 & 12394.97 & 32 & 12394.97 & 26 & 31 & lower secondary & teacher & industry \\
\hline 1105 & 12554.55 & 33 & 13148.84 & 31 & 32 & primary & blue collar & industry \\
\hline 2 & 12743.68 & 34 & 12743.68 & 29 & 33 & primary & self-employed & public administ \\
\hline 3 & 12894.1 & 35 & 12646.49 & 27 & 34 & primary & jnr manager-official & private services \\
\hline 275 & 13358.67 & 36 & 14304.81 & 35 & 35 & primary & blue collar & public administ \\
\hline 382 & 13530.88 & 37 & 14775.02 & 39 & 36 & lower secondary & office worker & private services \\
\hline 2 & 13696.73 & 38 & 13696.73 & 32 & 37 & no educ & entrepreneur & industry \\
\hline 553 & 13753.49 & 39 & 14622.13 & 37 & 38 & lower secondary & blue collar & public administ \\
\hline 858 & 13784.59 & 41 & 18956.16 & 68 & 39 & upper secondary & self-employed & private services \\
\hline 13 & 13912.12 & 42 & 12081.74 & 22 & 40 & no educ & blue collar & public administ \\
\hline 1364 & 13944.34 & 44 & 15911.1 & 47 & 41 & upper secondary & office worker & private services \\
\hline 65 & 13944.34 & 44 & 19831.28 & 73 & 42 & upper secondary & self-employed & agriculture \\
\hline 220 & 13949.5 & 46.5 & 14935.23 & 40 & 43 & upper secondary & blue collar & public administ \\
\hline 871 & 13949.5 & 46.5 & 15199 & 42 & 44 & upper secondary & teacher & public administ \\
\hline 45 & 13975.32 & 48 & 15005.54 & 41 & 45 & primary & office worker & private services \\
\hline 322 & 13990.24 & 49 & 17233.78 & 52 & 46 & primary & self-employed & industry \\
\hline 446 & 14066.39 & 50 & 18093.11 & 61 & 47 & lower secondary & self-employed & industry \\
\hline 11 & 14090.41 & 51 & 16717.96 & 50 & 48 & bachelor & teacher & private services \\
\hline
\end{tabular}




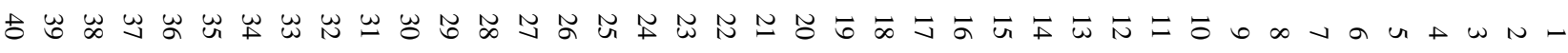

Table 7. Continued.

\begin{tabular}{|c|c|c|c|c|c|c|c|c|}
\hline Cases & $\begin{array}{c}\text { Median } \\
\text { income } \\
\text { (1998 euros) }\end{array}$ & $\begin{array}{l}\text { Rank } \\
\text { median }\end{array}$ & $\begin{array}{c}\text { Mean } \\
\text { income } \\
\text { (1998 euros) }\end{array}$ & $\begin{array}{l}\text { Rank } \\
\text { mean }\end{array}$ & $\begin{array}{l}\text { Rank } \\
\text { (final) }\end{array}$ & Education & Work status & Sector of activity \\
\hline 44 & 14128.93 & 52 & 18400.5 & 64 & 49 & upper secondary & office worker & agriculture \\
\hline 17 & 14212.86 & 53 & 17509.45 & 54 & 50 & upper secondary & self-employed & public administ \\
\hline 118 & 14290.47 & 54 & 15252.5 & 43 & 51 & primary & office worker & public administ \\
\hline 36 & 14361.15 & 55 & 15869.1 & 46 & 52 & lower secondary & professional & private services \\
\hline 289 & 14460.79 & 56 & 19683.29 & 72 & 53 & upper secondary & self-employed & industry \\
\hline 26 & 14536.64 & 57 & 19343.26 & 71 & 54 & primary & office worker & industry \\
\hline 1768 & 14937.15 & 58 & 16554.51 & 49 & 55 & upper secondary & office worker & public administ \\
\hline 94 & 15404.68 & 59 & 19857.04 & 74 & 56 & bachelor & office worker & industry \\
\hline 281 & 15406.18 & 60 & 17534.88 & 55 & 57 & bachelor & office worker & public administ \\
\hline 1008 & 15469.01 & 61 & 17832.16 & 58 & 58 & upper secondary & office worker & industry \\
\hline 2 & 15476.17 & 62 & 15476.17 & 44 & 59 & no educ & office worker & public administ \\
\hline 975 & 15493.71 & 63 & 17555.64 & 56 & 60 & bachelor & teacher & public administ \\
\hline 2 & 15686.32 & 64 & 15686.32 & 45 & 61 & primary & snr manager & private services \\
\hline 20 & 12043.38 & 29 & 18435.09 & 65 & 62 & lower secondary & self-employed & public administ \\
\hline 29 & 16041.54 & 65 & 25192.92 & 89 & 63 & primary & entrepreneur & industry \\
\hline 250 & 16306.92 & 66 & 17835.67 & 59 & 64 & lower secondary & office worker & industry \\
\hline 12 & 16540.43 & 67 & 21193.03 & 79 & 65 & upper secondary & professional & public administ \\
\hline 291 & 16547.6 & 68 & 20999.01 & 78 & 66 & upper secondary & professional & private services \\
\hline 849 & 16678.25 & 69 & 17680.16 & 57 & 67 & lower secondary & office worker & public administ \\
\hline 32 & 13765.8 & 40 & 19154.38 & 70 & 68 & lower secondary & entrepreneur & industry \\
\hline 2 & 16919.13 & 70 & 16919.13 & 51 & 69 & lower secondary & professional & agriculture \\
\hline 156 & 17030.73 & 71 & 18991.5 & 69 & 70 & bachelor & office worker & private services \\
\hline 6 & 17148.23 & 72 & 17412.18 & 53 & 71 & primary & professional & agriculture \\
\hline 20 & 17692.01 & 73 & 20245.8 & 75 & 72 & bachelor & self-employed & public administ \\
\hline
\end{tabular}




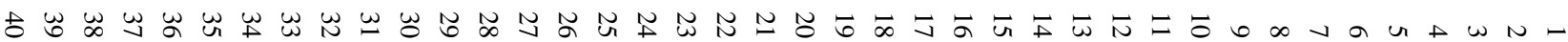

Table 7. Continued.

\begin{tabular}{|c|c|c|c|c|c|c|c|c|}
\hline Cases & $\begin{array}{c}\text { Median } \\
\text { income } \\
\text { (1998 euros) }\end{array}$ & $\begin{array}{l}\text { Rank } \\
\text { median }\end{array}$ & $\begin{array}{c}\text { Mean } \\
\text { income } \\
\text { (1998 euros) }\end{array}$ & $\begin{array}{l}\text { Rank } \\
\text { mean }\end{array}$ & $\begin{array}{l}\text { Rank } \\
\text { (final) }\end{array}$ & Education & Work status & Sector of activity \\
\hline 2 & 17985.93 & 74 & 17985.93 & 60 & 73 & lower secondary & snr manager & industry \\
\hline 52 & 18161.29 & 75 & 22066.41 & 85 & 74 & lower secondary & entrepreneur & private services \\
\hline 1 & 18174.67 & 76 & 18174.67 & 62 & 75 & upper secondary & entrepreneur & public administ \\
\hline 1 & 18305.3 & 77 & 18305.3 & 63 & 76 & upper secondary & teacher & agriculture \\
\hline 3 & 18417.79 & 78 & 15982.01 & 48 & 77 & primary & jnr manager-official & public administ \\
\hline 2 & 18506.64 & 79 & 18506.64 & 66 & 78 & primary & snr manager & industry \\
\hline 87 & 13944.34 & 44 & 21408.16 & 80 & 79 & bachelor & self-employed & private services \\
\hline 2 & 18696.55 & 80 & 18696.55 & 67 & 80 & upper secondary & teacher & industry \\
\hline 8 & 19290.46 & 81 & 42887.07 & 110 & 81 & primary & professional & private services \\
\hline 65 & 19358.99 & 82 & 21441.47 & 81 & 82 & lower secondary & jnr manager-official & public administ \\
\hline 71 & 19919.96 & 83 & 21762.98 & 83 & 83 & upper secondary & entrepreneur & private services \\
\hline 240 & 19993.02 & 84 & 22118.19 & 86 & 84 & upper secondary & jnr manager-official & public administ \\
\hline 9 & 20138.97 & 85 & 25958.64 & 90 & 85 & lower secondary & entrepreneur & agriculture \\
\hline 33 & 20563.59 & 86 & 21875.45 & 84 & 86 & lower secondary & jnr manager-official & private services \\
\hline 4 & 20585.48 & 87 & 28815.16 & 98 & 87 & bachelor & professional & agriculture \\
\hline 4 & 21335.5 & 88 & 20757.24 & 77 & 88 & upper secondary & professional & agriculture \\
\hline 2 & 21565.64 & 89 & 21565.64 & 82 & 89 & primary & professional & public administ \\
\hline 8 & 21849.42 & 90 & 26182.05 & 92 & 90 & lower secondary & professional & industry \\
\hline 8 & 21995.49 & 91 & 22292.86 & 87 & 91 & bachelor & office worker & agriculture \\
\hline 5 & 22968.3 & 92 & 20658.65 & 76 & 92 & upper secondary & jnr manager-official & agriculture \\
\hline 157 & 22985.43 & 93 & 25978.43 & 91 & 93 & upper secondary & jnr manager-official & industry \\
\hline 73 & 23091.15 & 94 & 32513.75 & 101 & 94 & bachelor & professional & industry \\
\hline 11 & 23347.98 & 95 & 37505.47 & 106 & 95 & primary & entrepreneur & agriculture \\
\hline 14 & 23457.87 & 96 & 32679.72 & 102 & 96 & upper secondary & entrepreneur & agriculture \\
\hline 23 & 23918.16 & 97 & 27732.29 & 94 & 97 & lower secondary & jnr manager-official & industry \\
\hline
\end{tabular}




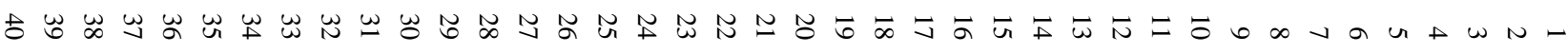

Table 7. Continued.

\begin{tabular}{|c|c|c|c|c|c|c|c|c|}
\hline Cases & $\begin{array}{c}\text { Median } \\
\text { income } \\
\text { (1998 euros) }\end{array}$ & $\begin{array}{c}\text { Rank } \\
\text { median }\end{array}$ & $\begin{array}{c}\text { Mean } \\
\text { income } \\
\text { (1998 euros) }\end{array}$ & $\begin{array}{l}\text { Rank } \\
\text { mean }\end{array}$ & $\begin{array}{l}\text { Rank } \\
\text { (final) }\end{array}$ & Education & Work status & Sector of activity \\
\hline 71 & 24505.05 & 98 & 24313.06 & 88 & 98 & upper secondary & professional & industry \\
\hline 252 & 24978.95 & 99 & 39702.71 & 108 & 99 & bachelor & professional & private services \\
\hline 210 & 25194.76 & 100 & 27532.91 & 93 & 100 & upper secondary & jnr manager-official & private services \\
\hline 170 & 25721.15 & 101 & 28778.76 & 97 & 101 & bachelor & jnr manager-official & public administ \\
\hline 79 & 26006.46 & 102 & 29904.88 & 100 & 102 & bachelor & jnr manager-official & industry \\
\hline 23 & 26289.81 & 103 & 75293.87 & 119 & 103 & bachelor & self-employed & industry \\
\hline 33 & 27075.66 & 104 & 61512.33 & 117 & 104 & upper secondary & entrepreneur & industry \\
\hline 86 & 27384.52 & 105 & 36503.56 & 105 & 105 & bachelor & jnr manager-official & private services \\
\hline 46 & 27910.26 & 106 & 28076.23 & 95 & 106 & upper secondary & snr manager & public administ \\
\hline 2 & 28508.01 & 107 & 28508.01 & 96 & 107 & lower secondary & snr manager & public administ \\
\hline 108 & 28795.71 & 108 & 33401.12 & 103 & 108 & bachelor & professional & public administ \\
\hline 4 & 31171.61 & 109 & 29685.89 & 99 & 109 & bachelor & jnr manager-official & agriculture \\
\hline 1 & 34318.56 & 110 & 34318.56 & 104 & 110 & no educ & professional & agriculture \\
\hline 209 & 34460.43 & 111 & 39126.57 & 107 & 111 & bachelor & snr manager & public administ \\
\hline 60 & 42435.52 & 112 & 43358.65 & 111 & 112 & upper secondary & snr manager & private services \\
\hline 2 & 42783.27 & 113 & 42783.27 & 109 & 113 & upper secondary & snr manager & agriculture \\
\hline 40 & 43438.16 & 114 & 52591.92 & 115 & 114 & upper secondary & snr manager & industry \\
\hline 2 & 44032.52 & 115 & 44032.52 & 112 & 115 & primary & jnr manager-official & industry \\
\hline 8 & 47199.6 & 116 & 64738.52 & 118 & 116 & bachelor & entrepreneur & private services \\
\hline 42 & 47366.23 & 117 & 49192.59 & 114 & 117 & bachelor & snr manager & industry \\
\hline 2 & 48852.52 & 118 & 48852.52 & 113 & 118 & bachelor & snr manager & agriculture \\
\hline 44 & 49686.13 & 119 & 53298.87 & 116 & 119 & bachelor & snr manager & private services \\
\hline 3 & 64942.07 & 120 & 119733.4 & 120 & 120 & bachelor & entrepreneur & agriculture \\
\hline 3 & 71434.56 & 121 & 123497.5 & 121 & 121 & bachelor & entrepreneur & industry \\
\hline 1 & 189513.8 & 122 & 189513.8 & 122 & 122 & bachelor & self-employed & agriculture \\
\hline
\end{tabular}


Table 8. Inequality measures - Italy 1993, 1995, 1998.

\begin{tabular}{lccccccccccc}
\hline & \multicolumn{3}{c}{ Household head } & \multicolumn{3}{c}{ Spouse household head } & \multicolumn{2}{c}{$\begin{array}{c}\text { Household head } \\
\text { father }\end{array}$} & $\begin{array}{c}\text { Household head } \\
\text { mother }\end{array}$ \\
\cline { 2 - 11 } & $\begin{array}{c}\text { actual } \\
\text { income }\end{array}$ & $\begin{array}{c}\text { median } \\
\text { income }\end{array}$ & $\begin{array}{c}\text { social } \\
\text { prestige }\end{array}$ & $\begin{array}{c}\text { actual } \\
\text { income }\end{array}$ & $\begin{array}{c}\text { median } \\
\text { income }\end{array}$ & $\begin{array}{c}\text { social } \\
\text { prestige }\end{array}$ & $\begin{array}{c}\text { median } \\
\text { income }\end{array}$ & $\begin{array}{c}\text { social } \\
\text { prestige }\end{array}$ & $\begin{array}{c}\text { median } \\
\text { income }\end{array}$ & $\begin{array}{c}\text { social } \\
\text { prestige }\end{array}$ \\
\hline Relative mean deviation & 0.230 & 0.126 & 0.225 & 0.207 & 0.083 & 0.187 & 0.133 & 0.300 & 0.129 & 0.316 \\
Coefficient of variation & 0.860 & 0.423 & 0.559 & 0.647 & 0.275 & 0.483 & 0.454 & 0.812 & 0.329 & 0.835 \\
Standard deviation of logs & 0.613 & 0.320 & 0.611 & 0.688 & 0.248 & 0.593 & 0.359 & 0.971 & 0.321 & 0.970 \\
Gini coefficient & 0.330 & 0.182 & 0.306 & 0.307 & 0.128 & 0.265 & 0.198 & 0.423 & 0.177 & 0.439 \\
Mehran measure & 0.436 & 0.239 & 0.428 & 0.433 & 0.183 & 0.388 & 0.276 & 0.579 & 0.254 & 0.601 \\
Piesch measure & 0.278 & 0.154 & 0.245 & 0.244 & 0.101 & 0.204 & 0.159 & 0.345 & 0.138 & 0.357 \\
Kakwani measure & 0.103 & 0.035 & 0.084 & 0.091 & 0.019 & 0.068 & 0.040 & 0.160 & 0.030 & 0.173 \\
Theil entropy measure & 0.218 & 0.069 & 0.150 & 0.175 & 0.034 & 0.119 & 0.077 & 0.303 & 0.052 & 0.324 \\
Theil mean log deviation measure & 0.186 & 0.059 & 0.166 & 0.179 & 0.032 & 0.143 & 0.070 & 0.382 & 0.052 & 0.400 \\
Entropy measure GE-1 & 0.337 & 0.055 & 0.241 & 0.422 & 0.032 & 0.233 & 0.071 & 0.744 & 0.054 & 0.698 \\
\hline Number of observations & 11476 & 11476 & 11476 & 6676 & 6676 & 6676 & 10593 & 10593 & 3266 & 3266 \\
\hline
\end{tabular}

Note: "median income" corresponds to the median occupational income, reported in Table 7, Column 2; "social position" corresponds to the occupation ranking proposed in Table 7, Column 6. 
resources between its macro regions, with the southern regions having in general a lower level of socio-economic development. We consider then 5 main macro regions, the Northeast, Northwest, Center, Southeast and Southwest with the islands (Sicily and Sardinia). In general, being the Northern regions far richer than the Southern ones, and having generally experienced even higher levels of economic growth in the post-war years, we expect that most socioeconomic indices of mobility will show a much greater level of structural mobility in the North rather than in the South. If it is also true that northern regions are more open to class exchanges than southern ones, then we expect than most mobility indices will display greater values for the Northern regions as compared to the Southern ones. However, given the generalized and nationwide post-war process of mass scholarization, we expect also that using education as status variable may give a different picture: this is so because mass scholarization implies a greater distance between fathers' and sons' marginal distributions in the South rather than the North (since sons in the South have comparable levels of educations than in the North even in the presence of an educational gap between northern and southern fathers). Thus, we expect that the different sensitivity of the various indices to differing marginal distribution will show up more when looking at educational rather than occupational mobility.

Table 9 reports both the value and the relative ordering of the 10 indices for the five macro regions. The upper part of the table uses fathers and sons median occupational income while the bottom part uses fathers and sons years of education as status variables. A glance at the table shows that the table confirms our expectations on regional mobility patterns: when occupational income is used as status variable, the northern regions seem to display unambiguously more mobility than the southern ones, while using education there seems to be an opposite pattern, but with less agreement between the indices, with the absolute indices giving a picture which is more similar to the picture emerging when using occupational income as status indicator than the picture emerging from ordinal indices.

We now move to our last analysis, that is, the study of the temporal evolution of occupational and educational mobility in Italy. To get an appreciation of what has happened to intergenerational mobility in Italy over time, we have divided the families into groups according to sons' birth five-year cohort. Figure 1 shows the evolution of occupational income and educational mobility for the ten indices for the eight age cohorts of the sons. A glance at Figure 1 gives a quite striking picture: while mobility seems to be decreasing over time when using the first four indices, exactly the opposite view emerges using the last six indices. This impression is confirmed by looking at the correlation 


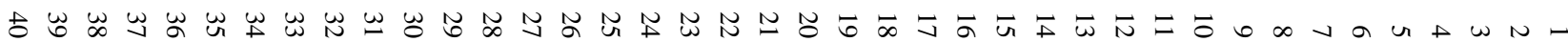

Table 9. Mobility measures - Italy 1993, 1995, 1998 - Regional Disaggregation.

\begin{tabular}{|c|c|c|c|c|c|c|c|c|c|c|c|}
\hline & \multicolumn{11}{|c|}{ Median Occupational Incomes } \\
\hline & obs & index 1 & index2 & index 3 & index 4 & index 5 & index6 & index7 & index 8 & index9 & index 10 \\
\hline Italy & 10593 & 4789 & 58700000 & 0.347 & 0.421 & 0.681 & 0.662 & 0.108 & 0.261 & 0.619 & 0.683 \\
\hline north-west & 2355 & 5300 & 75500000 & 0.343 & 0.469 & 0.742 & 0.674 & 0.112 & 0.269 & 0.685 & 0.694 \\
\hline north east & 2085 & 4956 & 77400000 & 0.350 & 0.569 & 0.743 & 0.753 & 0.121 & 0.280 & 0.661 & 0.736 \\
\hline center & 2346 & 4798 & 51600000 & 0.353 & 0.371 & 0.665 & 0.685 & 0.112 & 0.264 & 0.649 & 0.714 \\
\hline south-east & 1266 & 4521 & 45400000 & 0.358 & 0.374 & 0.614 & 0.604 & 0.097 & 0.247 & 0.513 & 0.644 \\
\hline \multirow{2}{*}{ south-west \& island } & 2541 & 4301 & 40800000 & 0.336 & 0.308 & 0.584 & 0.609 & 0.100 & 0.251 & 0.524 & 0.652 \\
\hline & avg. rank & rank1 & rank2 & rank3 & rank4 & rank5 & rank6 & rank7 & rank8 & rank9 & rank10 \\
\hline north-west & 4 & 5 & 4 & 2 & 4 & 4 & 3 & 4 & 4 & 5 & 3 \\
\hline north east & 5 & 4 & 5 & 3 & 5 & 5 & 5 & 5 & 5 & 4 & 5 \\
\hline center & 3 & 3 & 3 & 4 & 4 & 3 & 4 & 3 & 3 & 3 & 4 \\
\hline south-east & 2 & 2 & 2 & 5 & 3 & 2 & 1 & 1 & 1 & 1 & 1 \\
\hline south-west \& island & 1 & 1 & 1 & 1 & 1 & 1 & 2 & 2 & 2 & 2 & 2 \\
\hline
\end{tabular}




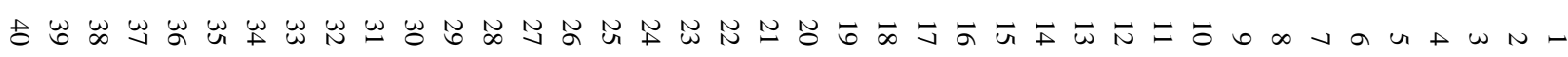

Table 9. Continued.

\begin{tabular}{|c|c|c|c|c|c|c|c|c|c|c|c|}
\hline & \multicolumn{11}{|c|}{ Years of Education } \\
\hline & obs & index 1 & index 2 & index3 & index 4 & index 5 & index6 & index7 & index 8 & index 9 & index 10 \\
\hline Italy & 11207 & 5.16 & 40.49 & 0.517 & 1.532 & 0.472 & 0.480 & 0.078 & 0.216 & 0.466 & 0.564 \\
\hline north-west & 2527 & 4.92 & 37.51 & 0.505 & 1.070 & 0.450 & 0.454 & 0.074 & 0.211 & 0.458 & 0.562 \\
\hline north east & 2170 & 5.12 & 39.97 & 0.524 & 1.471 & 0.533 & 0.540 & 0.089 & 0.232 & 0.516 & 0.587 \\
\hline center & 2472 & 5.34 & 42.23 & 0.539 & 1.631 & 0.515 & 0.526 & 0.086 & 0.228 & 0.532 & 0.622 \\
\hline south-east & 1334 & 5.40 & 43.57 & 0.530 & 2.105 & 0.458 & 0.462 & 0.073 & 0.209 & 0.433 & 0.557 \\
\hline \multirow[t]{2}{*}{ south-west \& island } & 2704 & 5.14 & 40.59 & 0.496 & 1.843 & 0.439 & 0.453 & 0.071 & 0.207 & 0.409 & 0.496 \\
\hline & avg. rank & rank1 & rank2 & rank3 & rank4 & rank5 & rank6 & rank7 & rank8 & rank9 & rank10 \\
\hline north-west & 2 & 1 & 1 & 2 & 1 & 2 & 2 & 3 & 3 & 3 & 3 \\
\hline north east & 4 & 2 & 2 & 3 & 2 & 5 & 5 & 5 & 5 & 2 & 4 \\
\hline center & 5 & 4 & 4 & 5 & 3 & 4 & 4 & 4 & 4 & 1 & 5 \\
\hline south-east & 4 & 5 & 5 & 4 & 5 & 3 & 3 & 2 & 2 & 4 & 2 \\
\hline south-west \& island & 2 & 3 & 3 & 1 & 4 & 1 & 1 & 1 & 1 & 5 & 1 \\
\hline
\end{tabular}



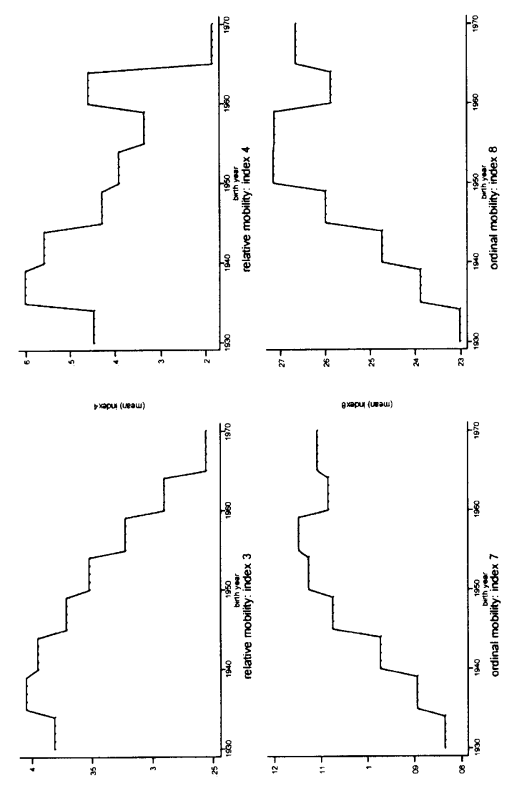

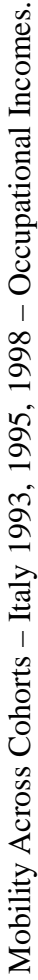

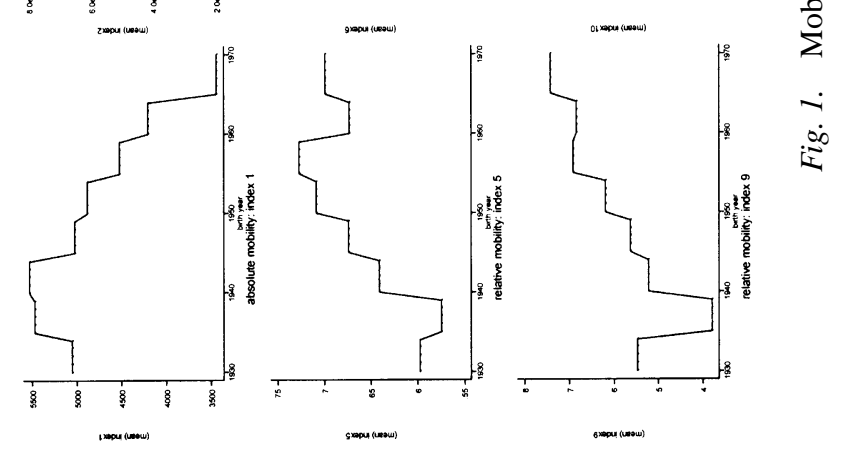




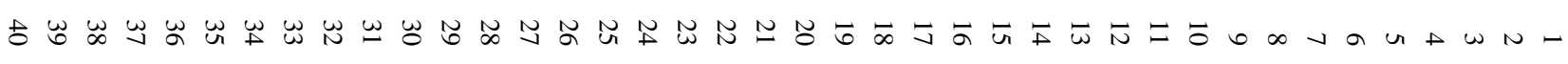

Table 10. Ordering of occupations - Italy 1993, 1995, 1998.

\begin{tabular}{|c|c|c|c|c|c|c|c|c|c|c|}
\hline & \multicolumn{10}{|c|}{ Median Occupational Incomes } \\
\hline & index 1 & index 2 & index 3 & index4 & index5 & index6 & index 7 & index 8 & index 9 & index 10 \\
\hline index1 & 1.0000 & & & & & & & & & \\
\hline index 2 & 0.7135 & 1.0000 & & & & & & & & \\
\hline index3 & 0.9843 & 0.6134 & 1.0000 & & & & & & & \\
\hline index4 & 0.8766 & 0.9368 & 0.8249 & 1.0000 & & & & & & \\
\hline index5 & -0.6056 & -0.6669 & -0.7249 & 1.0000 & & & & & & \\
\hline index6 & -0.6916 & -0.7557 & -0.7003 & 0.9472 & 1.0000 & & & & & \\
\hline index7 & -0.5923 & -0.6675 & -0.6285 & 0.9582 & 0.9884 & 1.0000 & & & & \\
\hline index8 & -0.5674 & -0.6321 & -0.6497 & 0.9590 & 0.9790 & 0.9904 & 1.0000 & & & \\
\hline index9 & -0.8772 & -0.9076 & -0.8613 & 0.8427 & 0.8076 & 0.7558 & 0.7277 & 1.0000 & & \\
\hline \multirow[t]{3}{*}{ index 10} & -0.7760 & -0.8130 & -0.8267 & 0.9459 & 0.9730 & 0.9386 & 0.9402 & 0.8802 & 1.0000 & \\
\hline & \multicolumn{10}{|c|}{ Years of Education } \\
\hline & index 1 & index 2 & index 3 & index4 & index 5 & index6 & index7 & index8 & index 9 & index 10 \\
\hline index 1 & 1.0000 & & & & & & & & & \\
\hline index2 & 0.9496 & 1.0000 & & & & & & & & \\
\hline index 3 & 0.9027 & 0.8089 & 1.0000 & & & & & & & \\
\hline index4 & 0.2718 & 0.5389 & -0.0225 & 1.0000 & & & & & & \\
\hline index 5 & 0.6941 & 0.4627 & 0.8107 & -0.4075 & 1.0000 & & & & & \\
\hline index6 & 0.5192 & 0.2412 & 0.6515 & -0.6132 & 0.9437 & 1.0000 & & & & \\
\hline index7 & 0.8308 & 0.6791 & 0.8659 & -0.1026 & 0.9194 & 0.8049 & 1.0000 & & & \\
\hline index8 & 0.7249 & 0.5195 & 0.8442 & -0.3577 & 0.9549 & 0.9271 & 0.9436 & 1.0000 & & \\
\hline index9 & 0.1582 & -0.1535 & 0.3610 & -0.8777 & -0.7619 & 0.8872 & 0.4951 & 0.6770 & 1.0000 & \\
\hline index10 & 0.0255 & -0.2433 & 0.3469 & -0.9053 & 0.6614 & 0.7488 & 0.3568 & 0.5596 & 0.9263 & 1.0000 \\
\hline
\end{tabular}


matrix between the indices in the upper part of Table 10, with the first four indices being all negatively correlated with the last six, and with high positive correlations within the two groups.

This strikingly different behavior of the various classes of mobility indices has again an explanation in the different weight given to the structural and exchange component of mobility by the different indices. In fact, given the decline of the rather fast industrialization process in Italy and the inverted Ushaped rate of growth of most post-war economic indicators (with exceptional growth rates until the mid 1970s and stagnation during the 1980s), structural mobility has been declining in the period of analysis, while changes in the openness of the society have caused an increase in exchange mobility.

Thus, we have two conflicting forces at work: fathers and sons marginal distributions have become "closer" over time (structural mobility has declined) while becoming also less positively associated (exchange mobility has increased). The net effect depends on the chosen class of indices. Looking at the temporal evolution of educational mobility gives a similar but less clear-cut picture, due to the different time it has required to close the educational gap between fathers and sons. It is worth noticing that both groups of indicators point to an increase of mobility for the generation born during the 1950s. This is probably entirely attributable to the massive educational reform introduced in 1960, which extended compulsory education from five to eight years and unified the lower secondary school. This educational push was at the same time an increase in absolute mobility (for educational reform was legally enforced, thanks to the construction of several new schools) and in relative mobility, because it allowed sons from lower family backgrounds to gain access to secondary education (poorer children were originally de facto discouraged by the existence of professional schools driving children from peasant families directly to work after five years of primary school).

\section{CONCLUSIONS}

Mobility data contain information of a very different nature: marginal distributions contain static information on the location and dispersion of status both in the fathers and sons generations; the distance between the fathers and sons marginal distributions gives information on the extent of structural mobility in the data; and the positive association between the two marginal distributions gives information on the openness of the society and the extent of its exchange mobility. Thus, comparing mobility data by a single summary 
mobility index is bound to give results that are very dependent on the characteristics of the chosen index.

This prediction is confirmed by our results. Indices that give relatively more weight to the structural component of mobility, may give a substantially different view than indices that give greater weight to the exchange component. For example, use of the first types of indices (absolute indices like $M_{1}$ or $M_{2}$ ) will result in arguing that intergenerational "mobility" is declining over time in post-war Italy while using ordinal indices (like $M_{5}$ or $M_{6}$ ) will give exactly the opposite impression.

A general teaching of this exercise is that intergenerational mobility is historically determined by the stage of development reached by a country. But this consideration suggests that cross-country comparisons in terms of intergenerational mobility (as we have done in our first exercise) have to be taken with caution, unless one can be sure that the countries considered have experienced similar patterns of socioeconomic growth. Being unable to control for the amount of structural mobility and using a single summary mobility index may render the conclusion reached tentative and very dependent on the chosen index.

It seems clear from our study that there is much scope for a clear formal definition of structural and exchange mobility and hence a decomposition of mobility indices into the separate contributions of the exchange and structural parts to overall mobility.

\section{NOTES}

1. See Fields 2001, Chapter 6 for an excellent discussion of various axioms that can be imposed on mobility indices.

2. With no ties, the difference lies in the fact that while $M_{6}$ divides the sum of the family difference in absolute ranks by $n^{3}, M_{7}$ divides by $n^{2}(n-1)$. Thus, in most cases the difference between the two indices is entirely due to the different treatment of tied ranks.

3. The countries are (in brackets the number of surveys): Australia (1), Brazil (2), Finland (1), Germany (8), Hungary (1), Indonesia (1), Ireland (1), Italy (1), Japan (1), Netherlands (4), Northern Ireland (2), Philippines (2), Switzerland (1), Taiwan (1), United Kingdom (2) and United States (2).

4. The panel component of the Bank of Italy survey of household wealth and income introduced was initially introduced in 1989 and subsequently expanded to one third of the sample in the following waves $(1991,1993,1995,1998)$.

5. See Barbagli et al. 1986.

6. A 65-year-old interviewee was 14 in 1934, while an 18-year-old interviewee was 14 in 1981. 
7. The original group of scholars used the occupational structure to construct a class structure, and analysed intergenerational mobility in terms of class mobility (Cobalti 1988; DeLillo, 1988; Schizzerotto, 1988; Barbagli, 1988; Cobalti-Schizzerotto, 1994; Schizzerotto-Bison, 1996). Mobility measure based on individual information (from the same data-set) can be found in Checchi-Ichino-Rustichini 1999.

8. For more detailed information see Brandolini 1999.

9. With reference to the 1985 survey on intergenerational mobility, DeLilloSchizzerotto 1985 have built an occupational prestige index of the reputational sort, i.e. interviewing a separate sample of individuals and asking them to rank a given number of occupations. Unfortunately there is no possibility to link this index with information available in the SHIW survey.

10. Duncan 1961 was the first one to propose an index of occupational prestige obtained as linear combination of these two variables. In general we must recall that reputational indices and incomes are not independently distributed (see Treiman, 1977). The Duncan index is constructed by giving half-weight to earnings; when constructing the Italian DeLillo-Schizzerotto index, the interviewees were asked to motivate the expressed ordering: the expected income in each occupation was indicated as the first reason for the proposed ordering.

11. Income data are converted in 1998 liras using the CPI inflation index, and then converted into euros to facilitate cross-country comparisons.

12. See Cannari-D' Alessio 1993 and Brandolini 1999.

13. The questionnaire asks "What were the educational qualifications, employment status and sector of activity of your parents when they were your current age?". This attenuates the "life-cycle bias" in measuring intergenerational mobility by keeping constant the age distance between parents and children. See Grawe 2001 for discussion of alternative research strategy on this issue.

14. The Pearson correlation coefficient is 0.93 , and the Spearman rank correlation coefficient is 0.94 .

15. However when the difference in ranking with the mean income exceeded a value of 30 positions (three cases in bold in Table 7), we have modified the relative ranking in accordance with the mean ranking.

16. The totals of table is lower than the totals of table because we impose the restriction of parents and children being contemporaneously employed.

17. Checchi, D'Agostino and Dardanoni (2001) consider the issue of marriage strategies and its effect on analyzing mobility using also information on mothers and daughters.

18. The territorial disaggregation could be distorted by different patterns of migration, occurred in Italy during the 1950s and the 1960s. However, taking the difference between the region of birth and the region of residence as a potential proxy for migration (and ignoring whether an individual experienced a period of migration out of the birth region), mobility measures are rather similar when either including or excluding permanent migrants.

\section{ACKNOWLEDGMENTS}

We thank an anonymous referee for helpful comments. We also thank $\mathrm{H}$. Ganzeboom and D. Treiman for kindly providing us with their dataset. 


\section{REFERENCES}

Barbagli, M. (1988). Da una classe all'altra. Polis- $\pi \circ \lambda_{\iota \sigma}, 1,5-18$.

Barbagli, M., Capecchi, V., Cobalti, A., de Lillo, A., \& Schizzerotto, A. (1986). Indagine nazionale sulla mobilià sociale: Computer file e documentazione.

Benabou, R., \& Ok, E. (2001). Mobility as progressivity: Ranking income processes according to equality of opportunity, NBER wp. 8431.

Brandolini, A. (1999). The distribution of personal income in post-war Italy: Source description, data quality, and the time pattern of income inequality. Bank of Italy Tema di Discussione No. 350 (April).

Cannari, L., \& D'Alessio, G. (1993). Non-reporting and under-reporting behavior in the bank of Italy's survey of household income and wealth. In: Proceedings of the ISI 49th Session (Florence), 395-412.

Checchi, D., Ichino, A., \& Rustichini, A. (1999). More equal but less mobile? Intergenerational mobility and inequality in Italy and in the U.S. Journal of Public Economics, 74, 351-393.

Checchi, D., D'Agostino, M., \& Dardanoni, V. (2001). The measurement of ordinal mobility: Theory and an application to Italy, paper presented to the SIEP annual conference (Pavia, September).

Cobalti, A. (1988). Mobili e diseguali. Polis- $\pi$ o $\iota \tau, 1,53-82$.

Cobalti, A., \& Schizzerotto, A. (1994). La mobilità sociale in Italia. Bologna: Il Mulino.

DeLillo, A. (1988). La mobilità sociale assoluta. Polis- $\pi \circ \lambda \iota \sigma, 1,19-52$.

DeLillo, A., \& Schizzerotto, A. (1985). La valutazione sociale delle occupazioni. Bologna: Il Mulino.

D'Agostino, M., \& Dardanoni, V. (2002). The measurement of mobility. Mimeo.

Duncan, O. (1961). A socioeconomic index for all occupations. In: A. Reiss (Ed.), Occupations and Social Status. New York: Free Press.

Fields, G. (2001). Distribution and Development. MIT Press.

Fields, G., \& Ok, E. (1996). The meaning and measurement of income mobility. Journal of Economic Theory, 71, 349-377.

Fields, G., \& Ok, E. (1999). Measuring movements in income. Economica, 66, 455-471.

Grawe, N. (2001). Quantile regression measures of mobility in the U.S. and abroad. Mimeo.

Kendall, M., \& Gibbons, J. (1990). Rank correlations methods. Edward Arnold.

Schizzerotto, A. (1988). Il ruolo dell'istruzione nei processi di mobilità sociale. Polis- $\pi \mathrm{o} \lambda \iota \sigma, 1$, $84-124$.

Schizzerotto, A., \& Bison, I. (1996). Mobilità occupazionale tra generazioni e mobilità di carriera: un confronto internazionale. In: G. Galli La mobilità della società italiana (pp. 445-508). Roma, SIPI.

Treiman, D. (1977). Occupational prestige in comparative perspective. New York: Academic Press.

Treiman, D., \& Ganzeboom, H. (1990). Cross-national comparative status attainment research, in AAVV. Research in Social Stratification and Mobility, 9, 105-127. JAI Press. 
